\title{
Approach-Phase Precision Landing with Hazard Relative Navigation: Terrestrial Test Campaign Results of the Morpheus/ALHAT Project
}

\author{
Timothy P. Crain* Robert H. Bishop $†$ John M. Carson III; \\ Nikolas Trawny, Chad Hanak, Jacob Sullivan, John Christian, \\ Kyle DeMars,* Tom Campbell, and Joel Getchius ${ }^{\dagger \dagger}$
}

The Morpheus Project began in late 2009 as an ambitious effort code-named Project M to integrate three ongoing multi-center NASA technology developments: humanoid robotics, liquid oxygen/liquid methane (LOX/LCH4) propulsion and Autonomous Precision Landing and Hazard Avoidance Technology (ALHAT) into a single engineering demonstration mission to be flown to the Moon by 2013. The humanoid robot effort was redirected to a deployment of Robonaut 2 on the International Space Station in February of 2011 while Morpheus continued as a terrestrial field test project integrating the existing ALHAT Project's technologies into a sub-orbital flight system using the world's first LOX/LCH4 main propulsion and reaction control system fed from the same blowdown tanks. A series of 33 tethered tests with the Morpheus 1.0 vehicle and Morpheus 1.5 vehicle were conducted from April 2011 - December 2013 before successful, sustained free flights with the primary Vertical Testbed (VTB) navigation configuration began with Free Flight 3 on December 10, 2013. Over the course of the following 12 free flights and 3 tethered flights, components of the ALHAT navigation system were integrated into the Morpheus vehicle, operations, and flight control loop. The ALHAT navigation system was integrated and run concurrently with the VTB navigation system as a reference and fail-safe option in flight (see touchdown position estimate comparisons in Fig. 1). Flight testing completed with Free Flight 15 on December 15,

*VP of Research \& Development, Intuitive Machines, Inc., 3700 Bay Area Blvd, Houston, TX 77058, tim@intuitivemachines.com

${ }^{\dagger}$ Professor, Electrical Engineering, University of South Florida, 4202 E. Fowler Ave.,Tampa, FL 33620, robertbishop@usf.edu

${ }^{\ddagger}$ ALHAT Deputy Lead, NASA Johnson Space Center (IPA Detail from NASA JPL), Houston, TX, 77058, john.m.carson@nasa.gov

§NASA Jet Propulsion Laboratory, Pasadena, CA, nikolas.trawny@jpl.nasa.gov

`Aerospace Engineer, NASA Johnson Space Center, Houston, TX, 77058, jacob.j.sullivan@nasa.gov

" Assistant Professor, Mechanical \& Aerospace Engineering, West Virginia University, Morgantown, WV 26506, john.christian@mail.wvu.edu

**Assistant Professor, Mechanical \& Aerospace Engineering, Missouri University of Science and Technology, 400 W. 13th Street, Rolla, MO 65409, demarsk@mst.edu

${ }^{\dagger}$ Senior Development Engineer, Intuitive Machines, Inc., 3700 Bay Area Blvd, Houston, TX 77058, joel.getchius@intuitivemachines.com 
2014 with a completely autonomous Hazard Detection and Avoidance (HDA), integration of surface relative and Hazard Relative Navigation (HRN) measurements into the onboard dual-state inertial estimator Kalman filter software, and landing within 2 meters of the VTB GPS-based navigation solution at the safe landing site target. This paper describes the Morpheus joint VTB/ALHAT navigation architecture, the sensors utilized during the terrestrial flight campaign, issues resolved during testing, and the navigation results from the flight tests.

The full ALHAT ${ }^{1-5}$ precision landing scenario considered a descent-to-landing from a parking orbit or a direct entry as illustrated in Fig. 2. The descent trajectory is a thrustcoast-thrust trajectory with high-altitude Terrain Relative Navigation (TRN) during the braking phase to correct for gross navigation errors relative to the planet and place the spacecraft within vicinity of the Intended Landing Point (ILP) determined from orbital surveys. After the braking phase, several surface relative sensors such as velocimeters, altimeters, and HRN would come online during the approach phase to provide for both soft touchdown capability as well as a locally precise landing within a small tolerance of a safe landing site. The safe landing site would be determined autonomously, in-flight by the ALHAT HDA system. ${ }^{6}$ The HRN sensor was dual use in that it formed the digital elevation map (DEM) used for safe site identification in the vicinity of the ILP and also tracked an automatically selected, distinct image patch "feature" registered within the DEM to provide sensor information to the Kalman filter. ${ }^{7}$ The integrated approach phase autonomous site selection, HRN feature tracking and precision navigation were the chosen for the Morpheus terrestrial demonstration objectives since the TRN capability had been previously demonstrated through techniques such as terrain correlation matching (TERCOM) and optical feature tracking on previous flight tests. ${ }^{8}$ Therefore, the Morpheus terrestrial flights included a boost phase from a launch pad that would be used to position the vehicle within the early stages of the approach phase to begin a simulated lunar landing.

The sensors used during Morpheus flight testing for the terrestrial flight campaign in the VTB navigation system included a GPS receiver, various inertial measurement units (IMUs), pre-flight optical attitude alignment ground support equipment, and an industrial laser range finder repurposed as an altimeter. The ALHAT navigation system was synchronized to the VTB navigation system prior to liftoff and processed information from the NASA Langley Research Center developed long range laser altimeter and doppler laser velocimeter, the Jet Propulsion Labs HRN sensor (based on a gimbaled flash lidar camera), and the same IMUs that fed the VTB navigation system. The Morpheus Autonomous Flight Manager (AFM) monitored the difference between the VTB and ALHAT navigation solutions during flight and was managed which navigation system was primary for flight control. The AFM implementation allowed the ALHAT navigation solution to be tested "open-loop" during early flights and fully integrated into the vehicle control path as sensor and navigation solution 
stability were demonstrated in flight. Both the VTB and ALHAT Kalman filters were based on an inertial dual-state formulation that traces its heritage to the Apollo and Space Shuttle programs that later became the common approach for the core of both the Orion and Morpheus navigation systems. This implementation is "dual-state" in that estimates of both the spacecraft and landing site inertial locations are included in the implementation so that the precision planetary landing navigation is very similar to chaser-target relative navigation during rendezvous. Additionally, the both of the Morpheus navigation implementations utilized a Markley Extended Kalman Filter (MEKF) ${ }^{9}$ approach for attitude estimation that was embedded within the same filter as the translation states. The care and feeding of the attitude state estimate within the coupled Kalman filter implementation was the source of significant effort during the Morpheus terrestrial flight testing campaign because the star trackers intended for use in the space application were not available for Earth testing. Eventually, a ground support equipment optical alignment technique ${ }^{10}$ was developed that allowed for the precision initialization of the VTB and ALHAT navigation systems and confirmation of maintained attitude state estimate quality prior to launch.

The organization of this paper is as follows: A description of the joint VTB/ALHAT navigation architecture is given with a focus on software partitioning and data interfaces. A discussion of the free flight mission profiles and the moding and processing of the various sensors on-board is presented to give operational context. The results from the VTB-only navigation flight tests are discussed. Issues encountered with integrating the ALHAT sensors for flight and during ALHAT flight testing are presented along with the solutions for these issues. The ALHAT HRN system integration with the overarching navigation software is described. A presentation and discussion of the final and successful VTB/ALHAT navigation systems' performance is given. Finally, a summary of the major results, conclusions, and recommendations for applying the Morpheus test results to space are presented. 


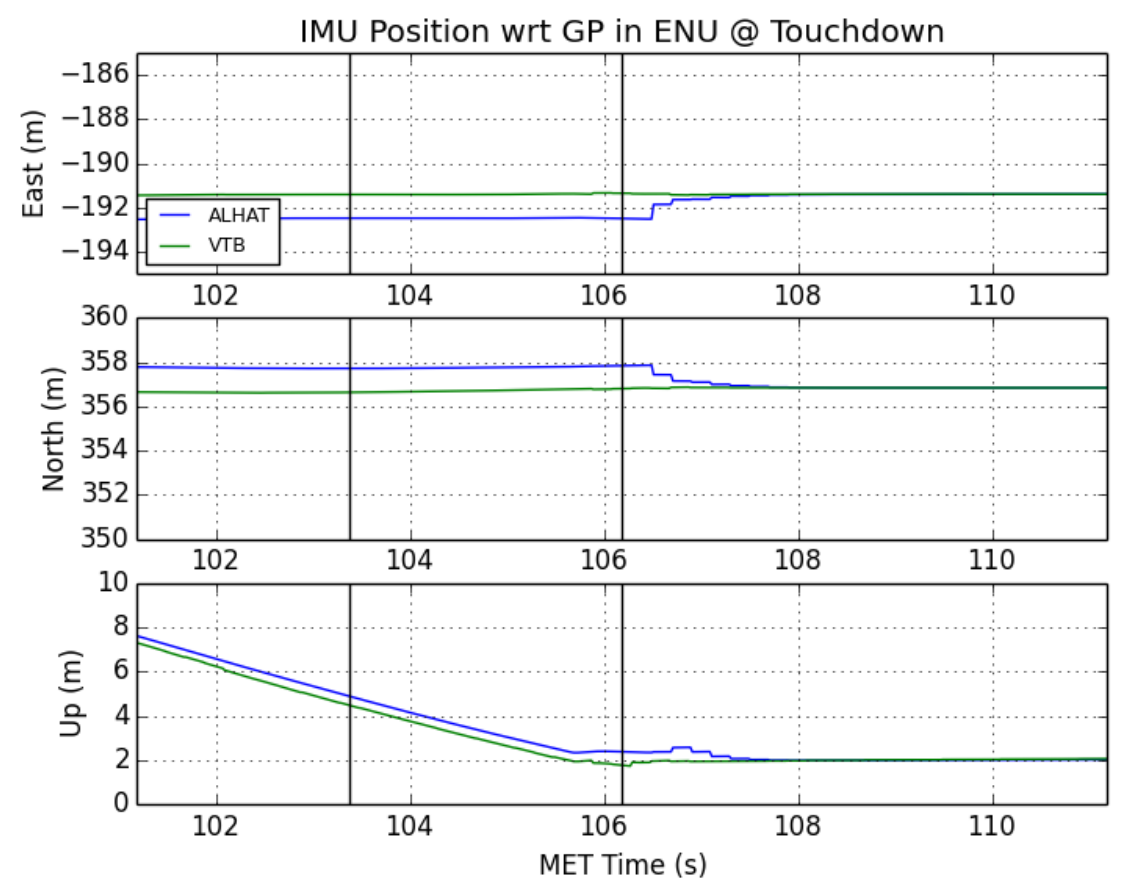

Figure 1. Free-Flight 15 VTB and ALHAT Position Estimate Comparisons Near Touchdown

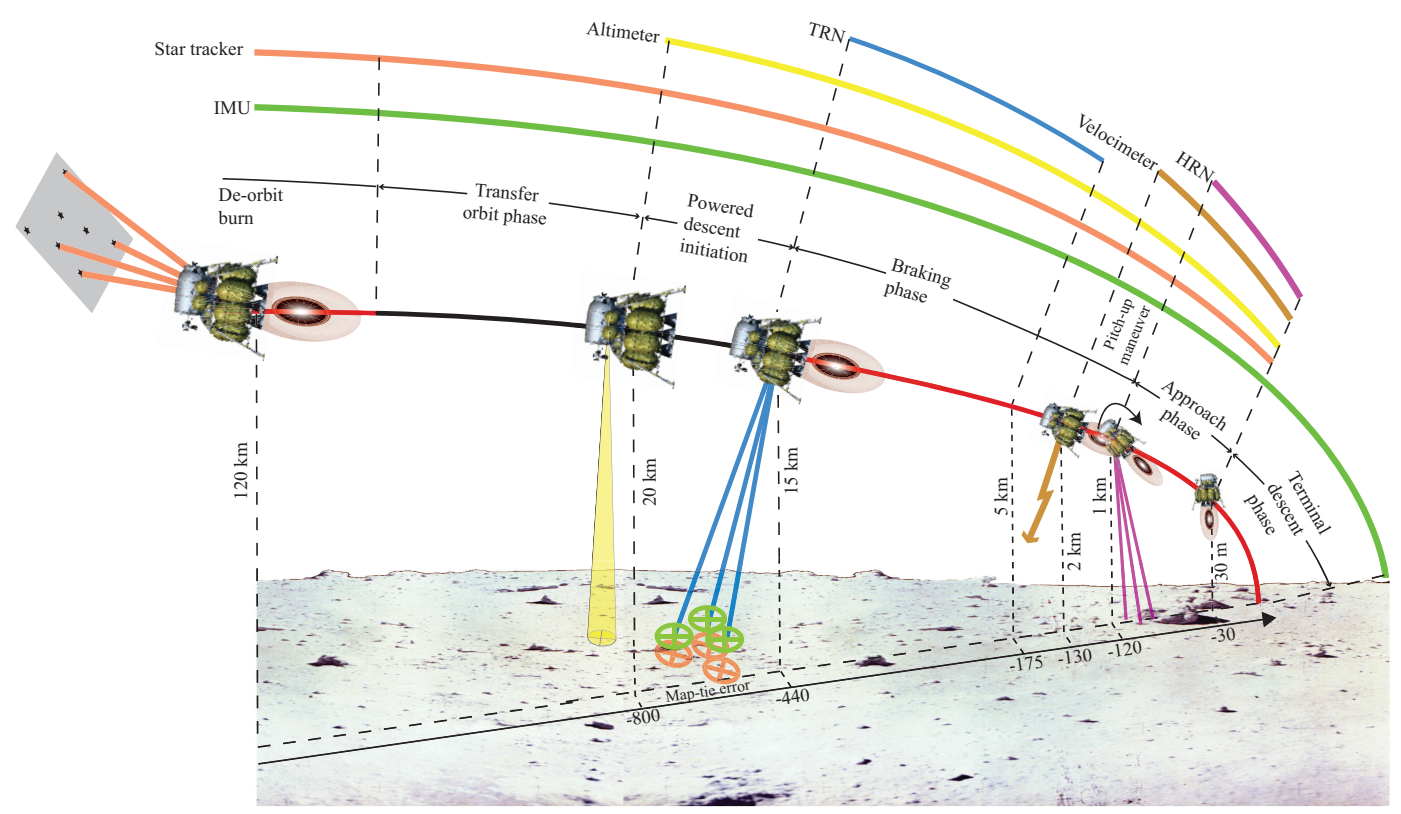

Figure 2. Schematic Descent-to-Landing Scenario 


\section{Navigation Architecture}

The Morpheus navigation design was required to support both stand-alone lander demonstrations as well as integrated test flights of the ALHAT technologies and algorithms. The architectural level navigation requirements for the joint VTB/ALHAT system are outlined in Table 1. The requirements Nav-1 and Nav-2 led to a split architecture implementation where common inertial navigation sensor data was used by the VTB and ALHAT strings of navigation as illustrated in Figure 3. The circular icons in this illustration are indicative of individual software applications with the following functions: I/O apps handling data collection and preliminary formatting from sensors, Kalman filter apps mechanizing an extended Kalman filter with specific measurement functions, fast propagation (fast prop) apps providing dynamic state updates between Kalman filter updates, and the user parameter processor (UPP) serving as the final clearing house for navigation data to all other applications and converted the internal navigation inertial translation and attitude states into rotating frame or target relative values. In order to efficiently support both strings of navigation; the IMU processing, system dynamics, and Kalman filter mechanization code was shared between both VTB and ALHAT. The differences in the two strings was manifested in the code for the measurement residual and partial derivatives for the specific measurements in the VTB and ALHAT strings.

Table 1. Morpheus VTB/ALHAT Navigation Architecture Requirements

\begin{tabular}{|c|l|}
\hline Reqt. ID & Requirement \\
\hline Nav-1 & $\begin{array}{l}\text { Provide VTB navigation as an in-flight comparison and down-select op- } \\
\text { tion when ALHAT was the primary navigation source. }\end{array}$ \\
\hline Nav-2 & $\begin{array}{l}\text { Incorporate only ALHAT technology navigation information into the } \\
\text { ALHAT Kalman filter (inertial measurements could be shared). }\end{array}$ \\
\hline Nav-3 & $\begin{array}{l}\text { Provide VTB navigation sufficient for landing on a 5m prepared landing } \\
\text { pad with a known ECEF location. }\end{array}$ \\
\hline Nav-4 & $\begin{array}{l}\text { Provide ALHAT navigation sufficient for landing on a prepared landing } \\
\text { pad within 3m of a target identified in-flight. }\end{array}$ \\
\hline
\end{tabular}

The inertial measurements were processed as delta velocity and delta attitude updates in an arbitrary inertial "IMU frame" established at navigation system initialization. The primary IMU (PIMU) used during Morpheus/ALHAT flight testing was a Honeywell Space Integrated GPS INS (SIGI) with an LN200 as backup IMU (BIMU) on the 1.0 and 1.5A vehicle through Free Flight 2 and a Systron-Donner 500 as BIMU on the 1.5B vehicle. The inertial book-keeping in flight software for both strings provided for an inflight switch from the PIMU to BIMU either from a detected fault in the PIMU or ground command. This capability was demonstrated both in simulation and during tethered flights (TT23 and TT24B).${ }^{11}$ Attitude initialization was achieved in a two step process which began with a 
gyro-compassing phase of up to 2 minutes which was found to converge within 0.01 degrees of local level (pitch and yaw of the vehicle with respect to local gravity) but was highly variable in the "roll" of the vehicle on the pad. The second step of attitude refinement utilized the Draper Labs GENIE Initial Direction Enhancer (GIDE) ${ }^{10}$ and is discussed in the ALHAT Integration section below. For both navigation strings, the common PIMU was used as the navigation base. The navigation accuracy requirements Nav-3 and Nav-4 influenced the type and precision of sensor measurements for the respective strings of navigation. This was particularly true for ALHAT navigation performance where the nature and operating range of each sensor was purposefully implemented after thorough analysis. ${ }^{12,13}$

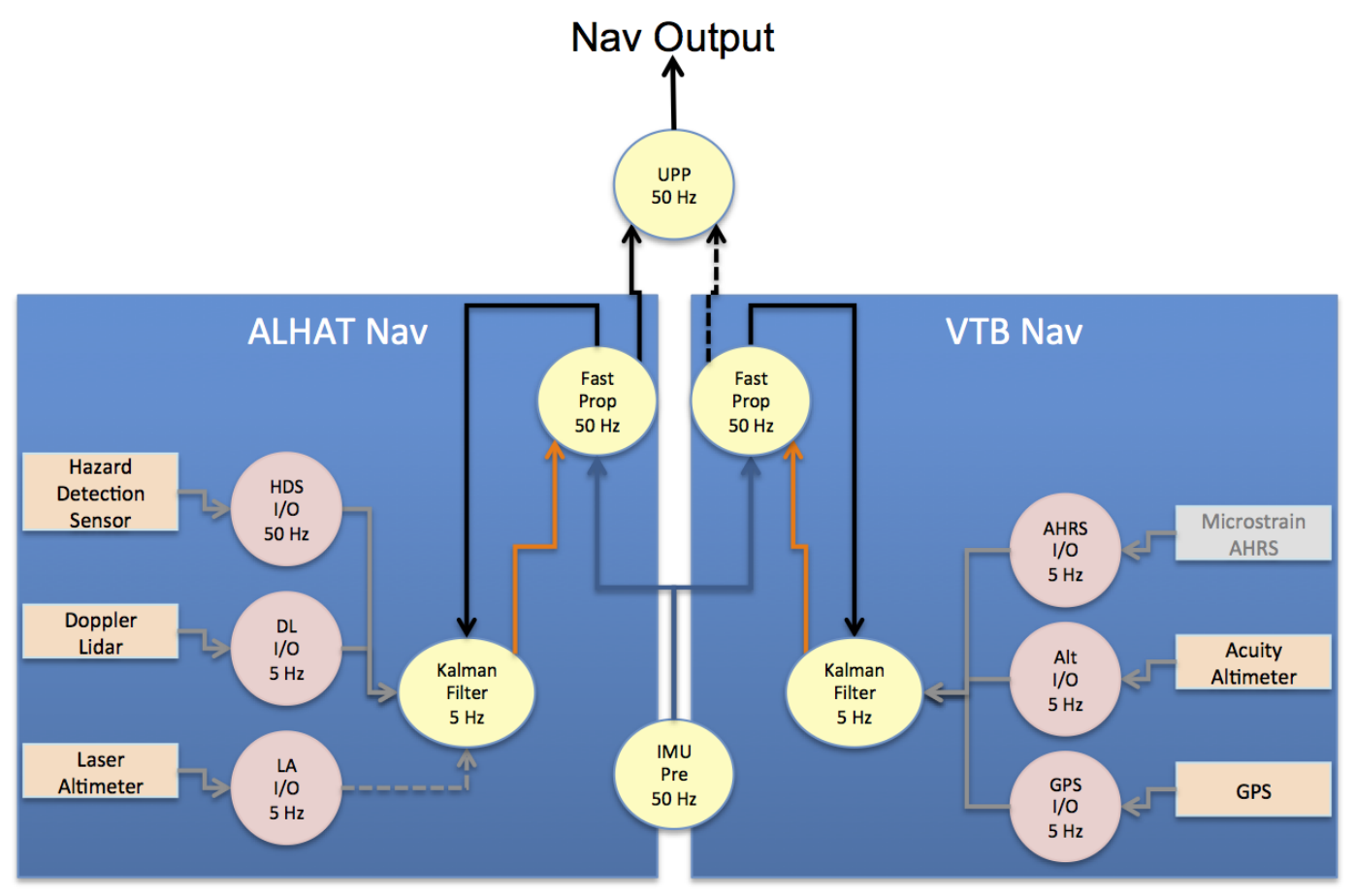

Figure 3. Dual Navigation System Architecture

\section{A. VTB Navigation}

The VTB navigation string incorporated Acuity laser range finder measurements as an altimeter via a slant range to a reference ellipsoid approximation and included Earth Centered Earth Fixed (ECEF) position and velocity measurements from a Javad GPS receiver. A Microstrain Attitude Heading Reference Sensor (AHRS) was integrated into the data collection as an independent reference but was not incorporated into VTB state updates. Flight testing of the AHRS proved that it was useful as a fault detection device before and after flight but it did not track navigation states well during powered flight. Representative measurement errors for the Acuity slant range and JAVAD GPS receiver position and velocity 
are provided in Table 2. The Acuity sensor operates in the near-infrared spectrum and was found to demonstrate a sensitivity to Morpheus propulsion system heating of the launch and landing pads as demonstrated in Free Flight 14 (FF14) around 20 seconds and 90 seconds in Figure 4. The navigation system proved robust to significant Acuity data dropouts from this effect.

Table 2. Morpheus VTB Observed Measurement Accuracies

\begin{tabular}{|c|c|c|c|c|}
\hline Measurement & Range & Bias & Noise & Drift over 60 Seconds \\
\hline Acuity Slant Range & $2-250 \mathrm{~m}$ & $<5 \mathrm{~cm}$ & $<1 \mathrm{~cm}$ & - \\
\hline JAVAD GPS Position & $\mathrm{n} / \mathrm{a}$ & $<2 \mathrm{~m}$ & $<2 \mathrm{~cm}$ & $<10 \mathrm{~cm}$ \\
\hline JAVAD GPS Velocity & $\mathrm{n} / \mathrm{a}$ & $<1 \mathrm{~cm} / \mathrm{s}$ & $<5 \mathrm{~cm} / \mathrm{s}$ & $<1 \mathrm{~cm} / \mathrm{s}$ \\
\hline
\end{tabular}
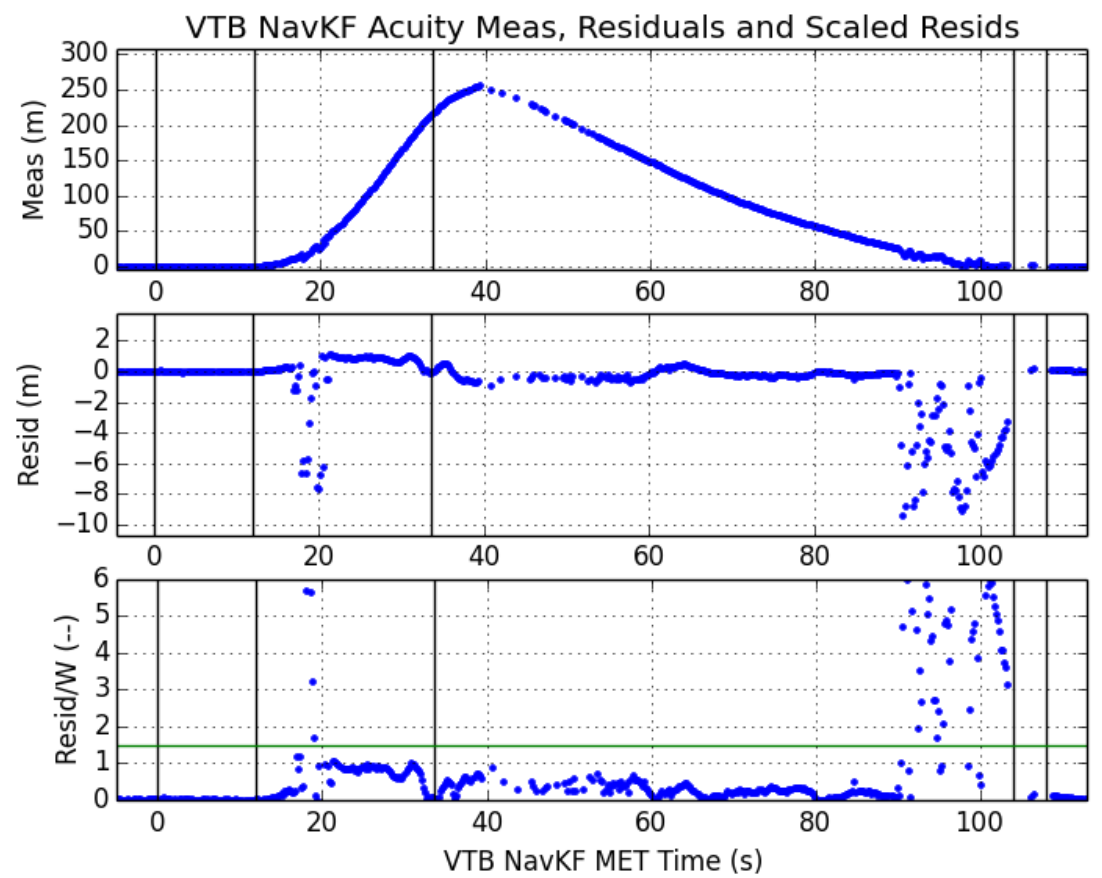

Figure 4. FF14 Acuity Altimeter Measurements, Pre-fit Residuals, and Residuals Scaled by Innovation Covariance $(W)$

\section{B. ALHAT Navigation}

The ALHAT navigation string incorporated measurements from the long-range Laser Altimeter (LA), Doppler Lidar (DL), and HDS/HRN sensors (see Figure 5) into its Kalman filter $^{4,5}$. All sensors were provided by the ALHAT NASA Langley Research Center team and the integration of the HRN sensor into the gimbal assembly and image processing avionics was performed by the ALHAT NASA Jet Propulsion Laboratory team. ${ }^{14}$ The LA measurements 
were functionally equivalent to the Acuity measurements and incorporated as a slant range to a reference ellipsoid. However, the LA produced three measurements per sample interval and the ALHAT filter only processed the last of the three. A sample of LA measurements from FF14 is provided in Figure 6. Like the Acuity sensor, the LA demonstrated a sensitivity to propulsion heating of the launch/landing platform. A side-by-side comparison of the third LA measurement per sample and the Acuity measurements for FF14 is provided in Figure 7.
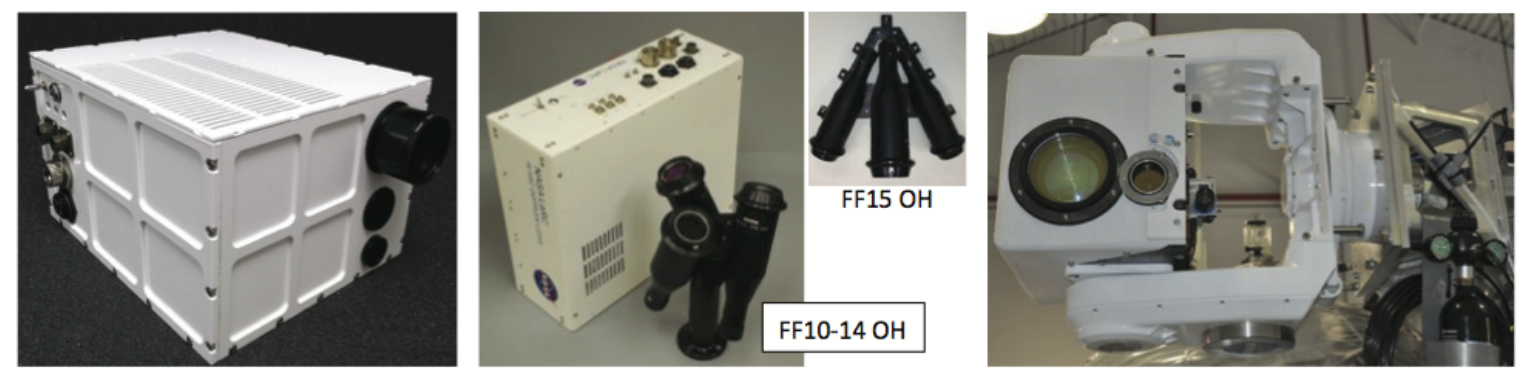

Figure 5. Prototype ALHAT sensors flight tested on Morpheus in 2014: LA (left), DL electronics and two versions of Optical Head (OH) (center), and HDS gimbaled flash lidar (right). ${ }^{6}$

The DL operated with a three-beam optical head providing line-of-sight slant range, line-of-sight slant range-rate, vector distance to the plane of beam intersection, and vector velocity with respect to the plane of intersection. However, only the line-of-sight range-rate measurements were processed in the ALHAT Kalman filter to limit the modeling requirements for measurement prediction in the flight software. The DL beams demonstrated a sensitivity to interaction with the heated column of air surrounding the Morpheus propulsion plume and provided a serious challenge to completion of the ALHAT navigation test objectives through FF14 as will be discussed later in this paper.

The HRN measurements were generated as part of the HDS which also created an inflight DEM of the landing area by with a gimbaled flash lidar sensor. The HDS identified an acceptable safe landing site within the DEM and then provided position estimates of the HDS sensor assembly with respect to a high-correlation feature in the DEM as a relative navigation cue for precision landing with respect to the safe landing site.

The ALHAT navigation string also processed a "navigation cross-feed" measurement prior to liftoff that fed the VTB navigation string inertial position and velocity solution to the ALHAT filter as a measurement. This was implemented to keep both navigation solutions synchronized prior to liftoff without unduly complicated initialization procedures prior to Morpheus launch. 

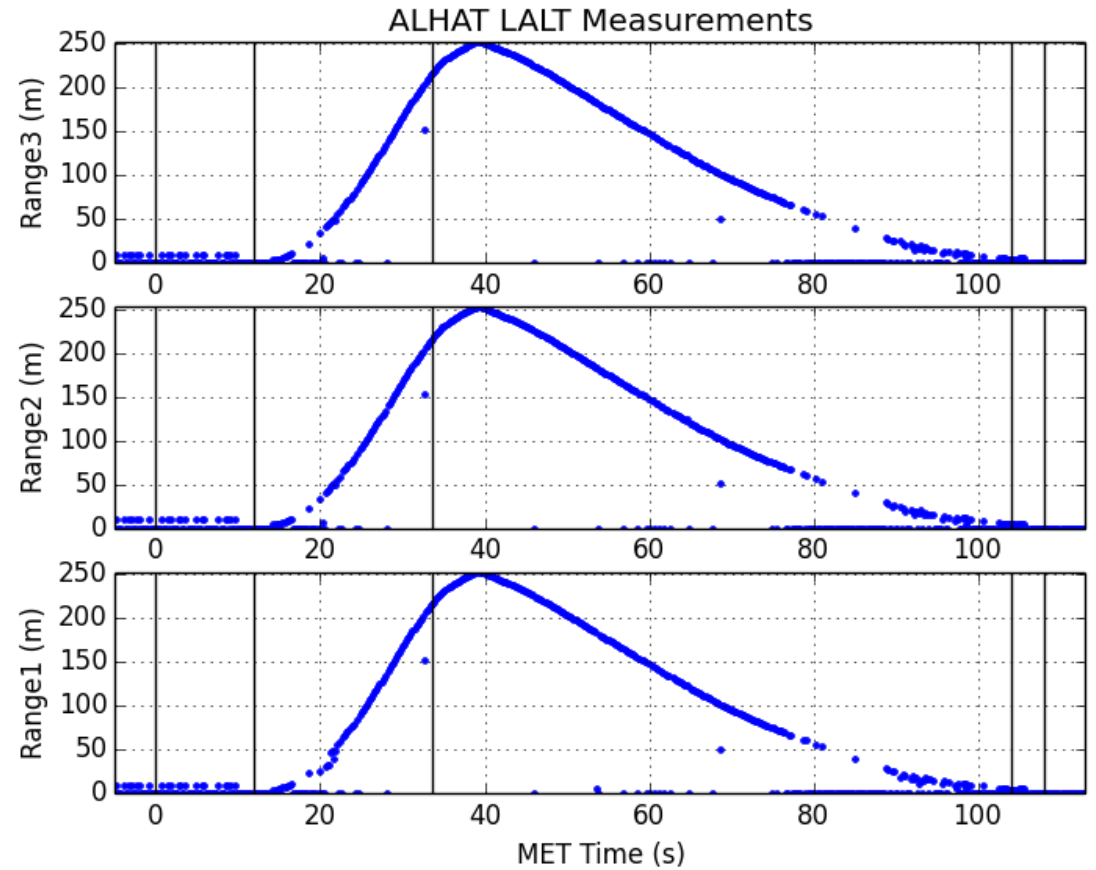

Figure 6. FF14 Laser Altimeter Measurements

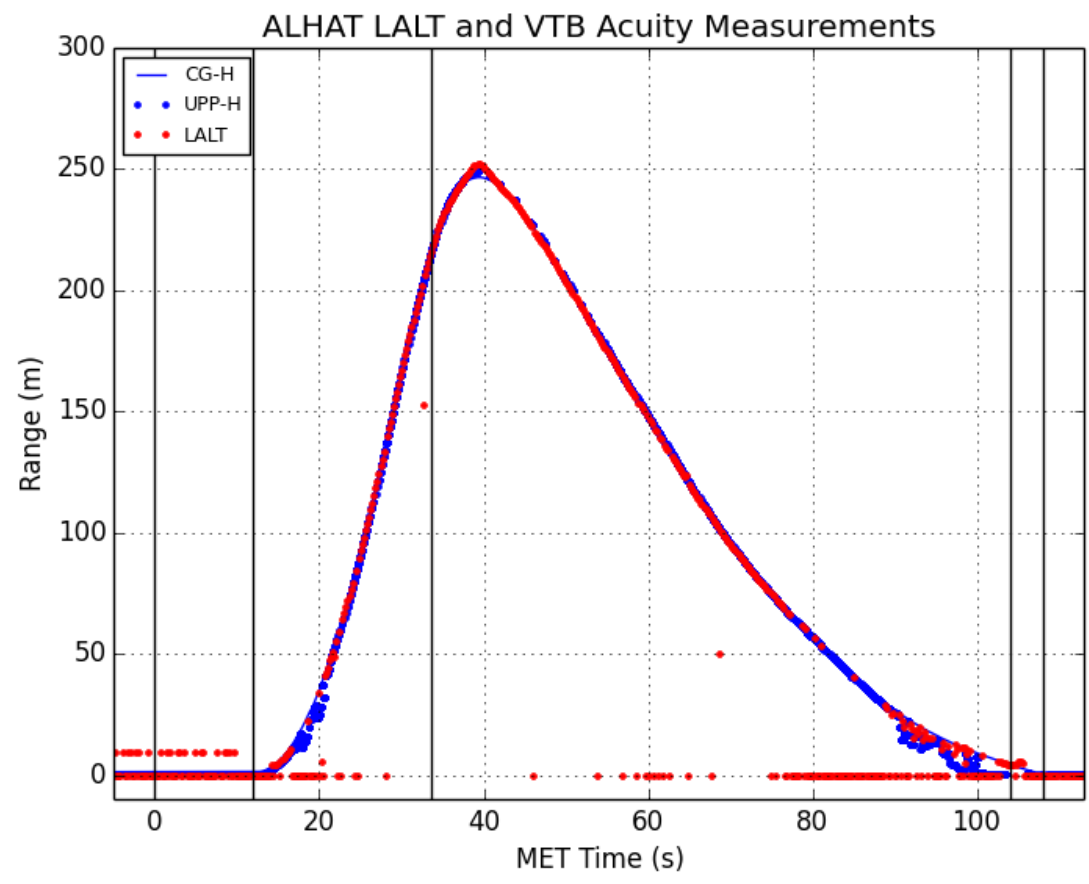

Figure 7. FF14 Laser Altimeter and Acuity Altimeter Measurements 
Table 3. Morpheus ALHAT Observed Measurement Accuracies

\begin{tabular}{|c|c|c|c|c|}
\hline Measurement & Range & Bias & Noise & Drift over 60 Seconds \\
\hline Laser Alt. Slant Range & $2-250 \mathrm{~m}$ & $<5 \mathrm{~cm}$ & $<1 \mathrm{~cm}$ & - \\
\hline Doppler Velocimetry & $\mathrm{n} / \mathrm{a}$ & $<1 \mathrm{~cm} / \mathrm{s}$ & $<3 \mathrm{~cm} / \mathrm{s}$ & - \\
\hline HDS Position & $\mathrm{n} / \mathrm{a}$ & $<2 \mathrm{~m}$ & - & - \\
\hline
\end{tabular}




\section{Mission Profile and Sensor Processing Schedule}

The Morpheus Project tested the ALHAT navigation technology for a simulated terminal phase of a lunar approach as illustrated in the final $1 \mathrm{~km}$ of Figure 2. A perspective view of this trajectory with the AFM soft-abort boundary prism is provided in Figure 8 . The flight path from the launch ground point (GP) to the ILP at the center of the simulated landing sight followed approximately a heading of 330 degrees at the end of the Shuttle Landing Facility (SLF) at the NASA Kennedy Space Center. The test trajectory began with a maximum acceleration boost phase to an altitude of approximately $250 \mathrm{~m}$ followed by a forward pitch to initiate downrange motion toward the center of the landing field at the ILP approximately 400m downrange. Typical trajectory position and velocity values from FF15 for ALHAT testing relative to the launch GP are provided in Figures 10 and 11. These results are presented in GP defined East-North-Up coordinates. The four vertical lines at approximately 12, 35, 102, and 105 seconds Mission Elapsed Time (MET) are key AFM mode transition markers as detailed in Table 4. MET on Morpheus flights begins with the initiation of Morpheus main stage ignition (with the exception of Free Flight 11).

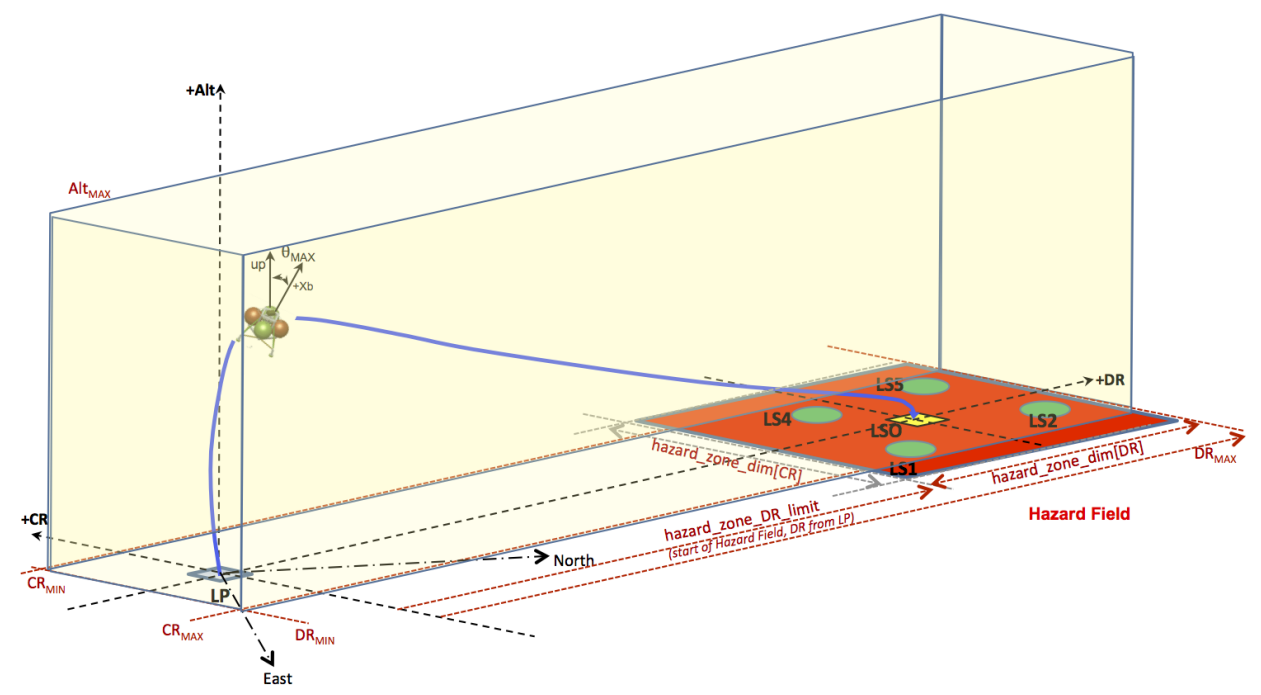

Figure 8. ALHAT Test Trajectory Perspective View

Table 4. Morpheus ALHAT Mode Transitions (Free Flight 15)

\begin{tabular}{|c|l|l|}
\hline MET & Mode Transition & Comment \\
\hline $12 \mathrm{~s}$ & Handover to onboard GNC for liftoff & Begin mostly vertical boost phase \\
\hline $35 \mathrm{~s}$ & Begin ALHAT HDS operations & During downrange motion to landing field \\
\hline $102 \mathrm{~s}$ & Terminal landing guidance logic arming & Approximately 5m above landing pad \\
\hline $105 \mathrm{~s}$ & Engine shutdown & Mission complete \\
\hline
\end{tabular}




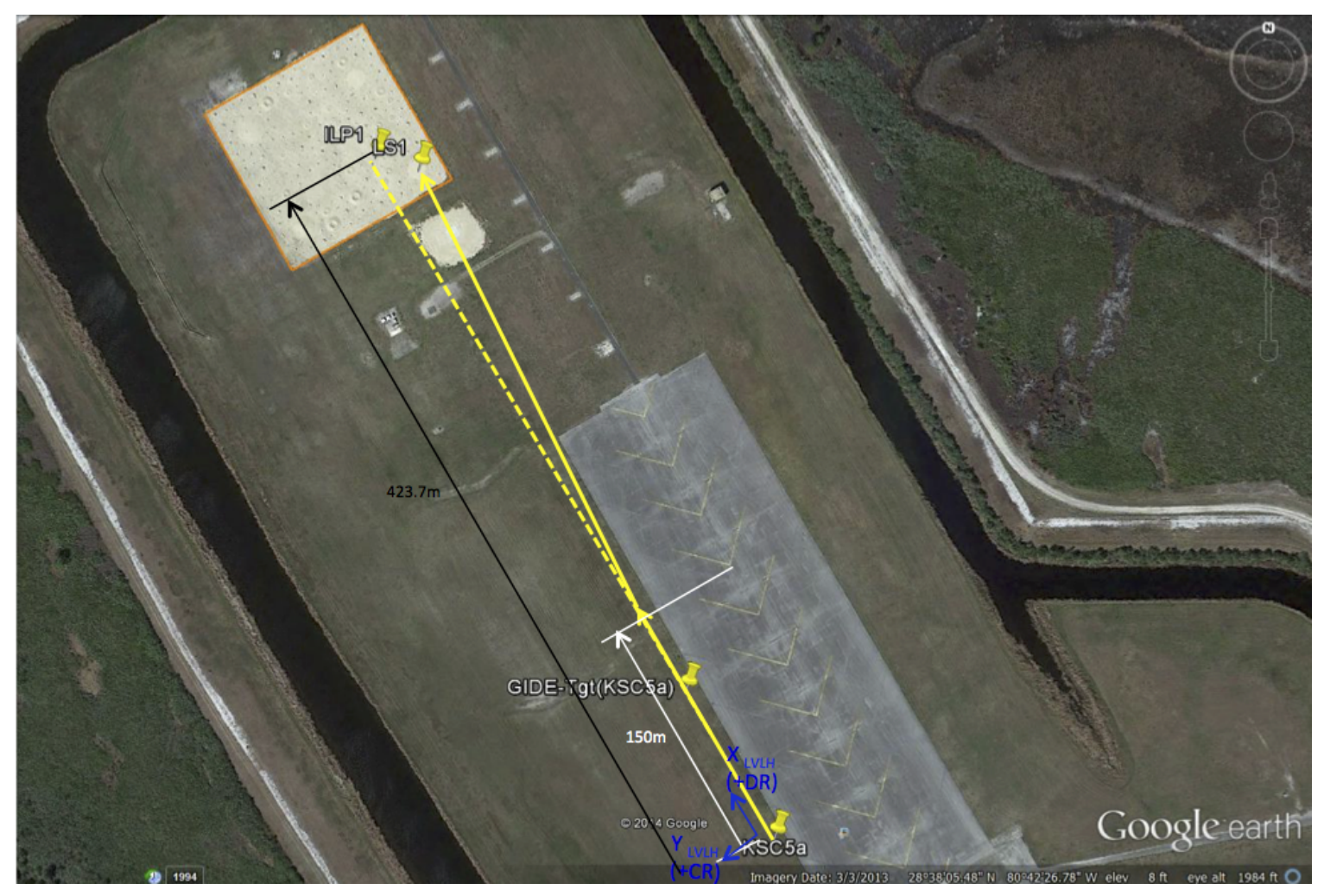

Figure 9. Configuration of the SLF Test Area for the Morpheus/ALHAT Flights ${ }^{5}$
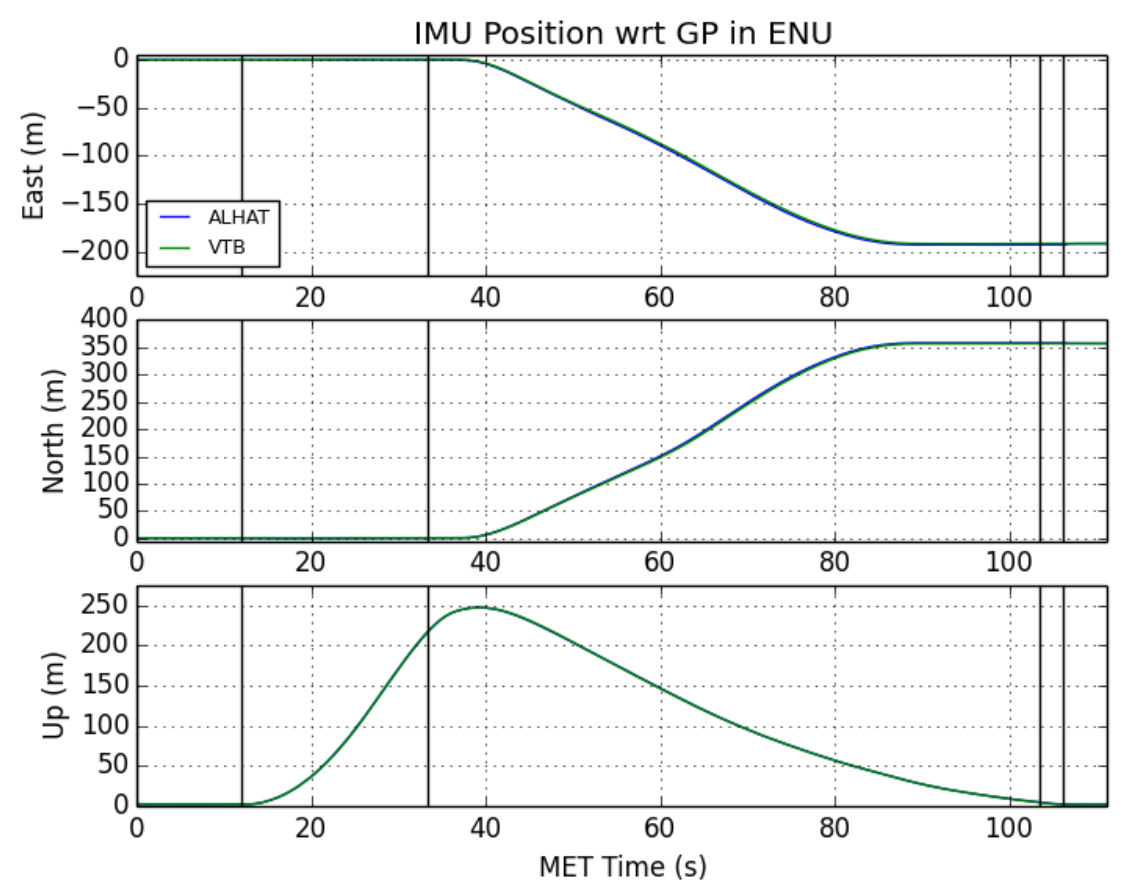

Figure 10. ALHAT Test Trajectory Position Histories (FF15 Results) 


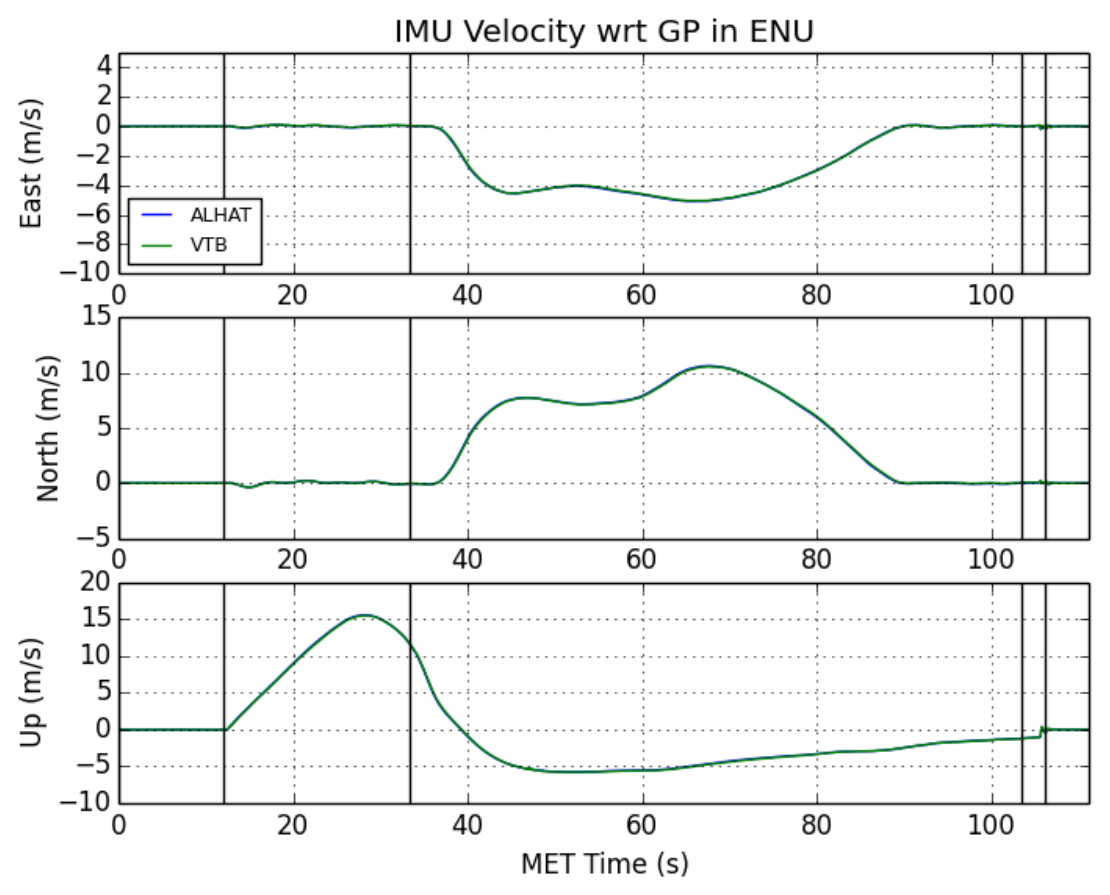

Figure 11. ALHAT Test Trajectory Velocity Histories (FF15 Results)

With two onboard navigation systems and a variety of sensors available, the AFM was required to manage the functionality of which navigation source was feeding the flight control loop, the sensors active to both navigation strings, navigation cross-feed activation/deactivation, and the navigation source providing inputs to the HDS gimbal steering controller. The system design documentation and operator control panels used the graphical key in Figure 12 to indicate the state of the navigation sources and active/available sensors (indicated with a filled green circle) throughout each phase of the Morpheus test flight. A nominal ALHAT test configuration sequence is depicted in Figure 13 where the ALHAT navigation string provides outputs for both the flight control and HDS gimbal control loops from liftoff to landing. The synchronizing VTB navigation cross-feed measurements were deactivated at ignition. The VTB navigation string was configured to process GPS position and velocity as well as Acuity slant range throughout flight. Initially, the DL velocimetry and LA slant range were active throughout the flight, but the ground heating interference with the LA measurements at landing led to the LA measurements eventually not being processed in final descent. HRN measurements were only enabled for the ALHAT filter in the HDS, approach, and approach_final phases of the Morpheus flight to align with the range at which the HDS laser sensor could provide meaningful measurements based on optical focus. In the event of a vehicle or ALHAT navigation string fault, AFM would switch the flight control navigation source to VTB but allow the ALHAT gimbal controls to continue 
to be fed by the ALHAT navigation string as depicted with a mid-flight fault scenario in Figure 14.

\begin{tabular}{|l|}
\hline Nav Src:ALHAT \\
\hline O GPS \\
O Acuity Alt \\
\hline O Gnd Pt \\
O HRN \\
O̊ DL-Vel \\
O DL-Alt \\
O LA-Alt \\
\hline \hline O VTB xfeed \\
\hline HDS UPP Src: \\
ALHAT \\
\hline
\end{tabular}

approach

Figure 12. Navigation Source and Processing Graph 


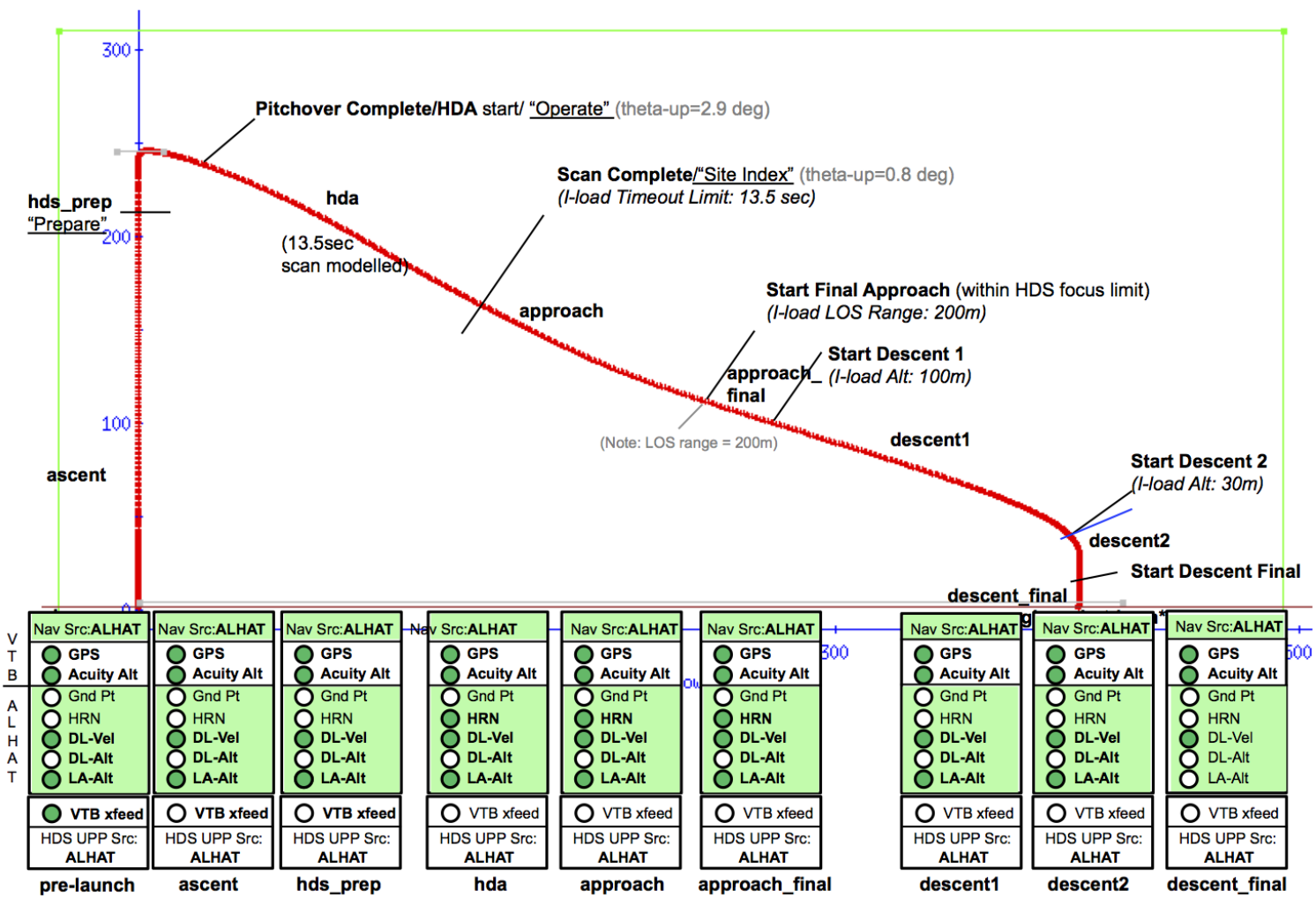

Figure 13. Nominal Sensor Processing Schedule

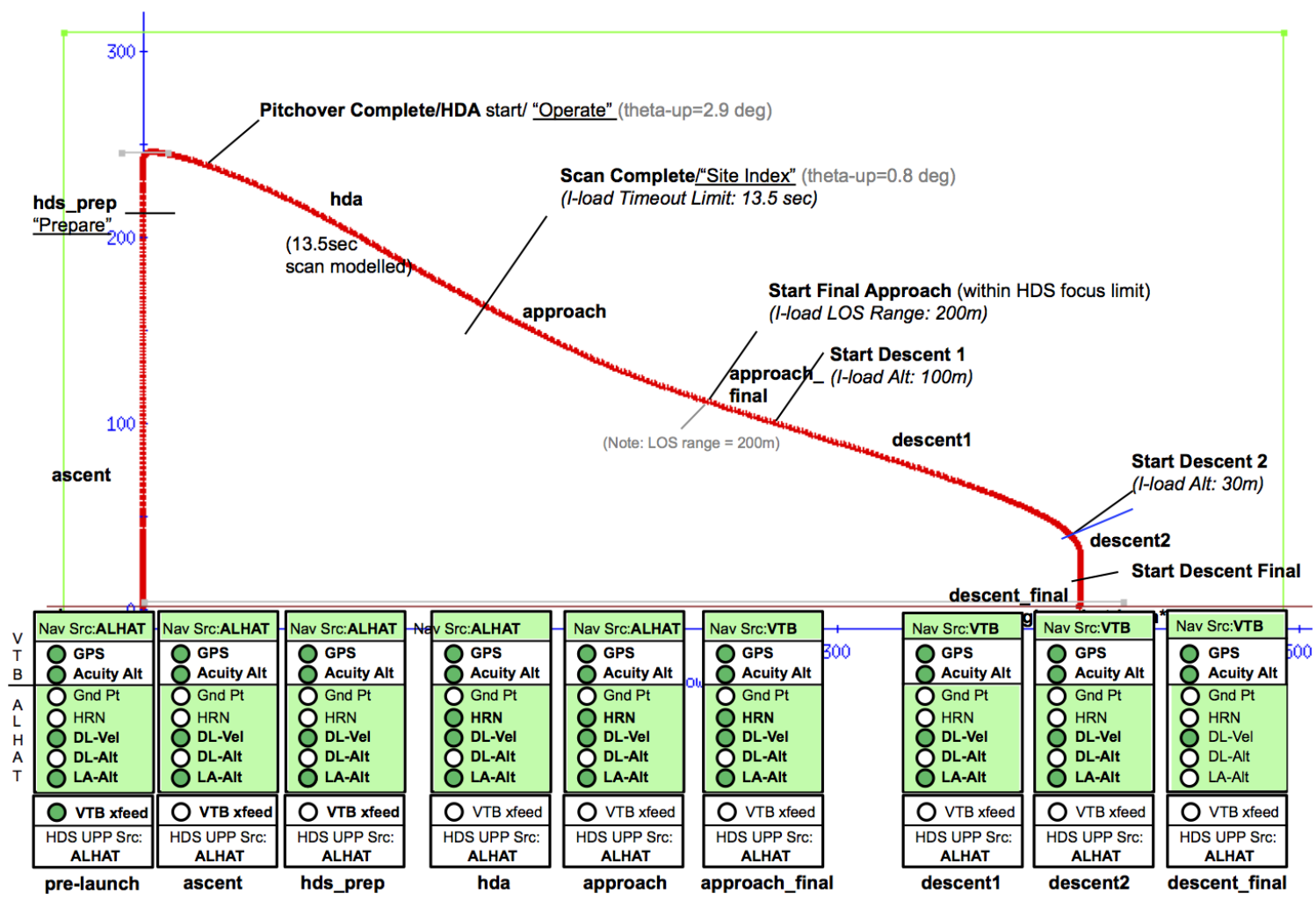

Figure 14. Fault-Down Sensor Processing Schedule 


\section{VTB-only Navigation Tests}

The Morpheus navigation team utilized every test and flight opportunity ${ }^{15}$ to evaluate, refine, and tune the operation of the VTB navigation system over a time period covering April 2011 to the final VTB-only navigation flight in March 2014 as outlined in Table 5. The significant bias in the GPS position (see Table 2) posed an early problem in that abort tolerances for VTB tethered tests and landing tolerances for ALHAT tests could easily be violated. A UPP resynchronization (UPP resynch) procedure was developed where the navigation system of both strings could be commanded to reset the GP ECEF location to be consistent with the current vehicle navigation state. In order to maintain consistency with the surveyed ECEF landing site locations, the adjustment applied to the GP was also equally applied to the landing site.

Table 5. VTB-only Navigation Test Flights

\begin{tabular}{|c|c|c|l|}
\hline Test & Date & Vehicle & Notes \\
\hline Hot Fire 1-2 & $4 / 2011$ & 1.0 & Environmental checkout \\
\hline \hline Tether Test 1 & $4 / 2011$ & 1.0 & Vehicle did not achieve flight \\
\hline Tether Test 2 & $4 / 2011$ & 1.0 & $\begin{array}{l}\text { Throttle failure, IMU orientation } \\
\text { error }\end{array}$ \\
\hline Tether Test 3-4 & $5 / 2011$ & 1.0 & Initial semi-stable flight \\
\hline Tether Test 5 & $6 / 2011$ & 1.0 & Stable flight, grass fire \\
\hline Tether Test 6 & $8 / 2011$ & 1.0 & Engine burn-through \\
\hline \hline Tether Test $7-15$ & $3 / 2012-5 / 2012$ & $1.5 \mathrm{~A}$ & Navigation characterization \\
\hline Tether Test 16-18 & $6 / 2012-7 / 2012$ & $1.5 \mathrm{~A}$ & ALHAT component testing \\
\hline Tether Test 19-20 & $7 / 2012-8 / 2012$ & $1.5 \mathrm{~A}$ & Free-flight tuning \\
\hline RCS Hot Fire 1 & $7 / 2012$ & $1.5 \mathrm{~A}$ & Fine rotation navigation demo \\
\hline Free Flight 1 & $8 / 2012$ & $1.5 \mathrm{~A}$ & Successful liftoff abort \\
\hline Free Flight 2 & $8 / 2012$ & $1.5 \mathrm{~A}$ & Loss of vehicle \\
\hline \hline Hot Fire 7-9 & $4 / 2013-5 / 2013$ & $1.5 \mathrm{~B}$ & Test on 1.5B \\
\hline Tether Test 21-22 & $5 / 2013-6 / 2013$ & $1.5 \mathrm{~B}$ & Resume flight operations \\
\hline Tether Test 23 & $6 / 2013$ & $1.5 \mathrm{~B}$ & $\begin{array}{l}\text { Full operations and flight on } \\
\text { backup IMU }\end{array}$ \\
\hline Tether Test 24A/B & $6 / 2013$ & $1.5 \mathrm{~B}$ & $\begin{array}{l}\text { Commanded down-mode to } \\
\text { backup IMU in flight }\end{array}$ \\
\hline Tether Test 25-33 & $7 / 2013-12 / 2013$ & $1.5 \mathrm{~B}$ & Guidance refinement \\
\hline Ground Takeoff/Landing & $11 / 2013$ & $1.5 \mathrm{~B}$ & Environment characterization \\
\hline Free Flight 3-9 & $12 / 2013-3 / 2014$ & $1.5 \mathrm{~B}$ & Envelope expansion \\
\hline & & & \\
\hline
\end{tabular}

\section{A. Initial Testing to Free Flight 2 Navigation Failure and Loss of Vehicle}

Tether Test 2 (TT2) was notable in that the Morpheus main engine throttle failed fully open prior to handover to the GNC system. On the same flight, a rotation of the PIMU 
internal sensing frame to the sensor case frame was not applied correctly in software resulting in a 90 degree rotation error of the incoming inertial data about the vehicle thrust axis. This orientation error did not manifest in Tether Test 1 because the vehicle did not attain flight and only through the navigation-to-control command chain was the error realized. In fact, extensive simulation of the tethered flight scenario did not reveal this error because the simulation was coded to the same interface control document (ICD) without the required addendum specifying the corrected rotation. Therefore, all simulated flights performed nominally. The rotation error proved fortuitous in TT2 in that it caused the GNC system to spin the vehicle and dissipated the thrust from the non-responsive throttle actuator until the emergency engine shut down command could be initiated remotely by the operations team. Both the throttle actuator and the navigation software were corrected after TT2 and successful, if semi-stable, flights were resumed within 5 days on TT3 and TT4. Based on the information gleaned from these initial tests, a full 34 second stable flight was achieved on Tether Test 5. However, the proximity of the Morpheus propulsion exhaust to the concrete test pad ejected superheated concrete fragments into the nearby grass causing a significant fire that led to modification of flight test procedures (raising the liftoff altitude, adding a burn break, etc.) and a stand-down of test operations for nearly 3 months. Other than the grass and some stored hay located nearby at the NASA Johnson Space Center, no damage was suffered to equipment or personnel by the grass fire which could only have been caused by a steady and well controlled hovering Morpheus.

Flight testing in the remainder of the tethered test campaign through TT20 and Free Flight 1 (FF1) was relatively uneventful from a navigation perspective and the data from these flights was used to build confidence in the VTB navigation system and characterize sensor performance. On Free Flight 2 (FF2), communications with the PIMU was lost approximately $300 \mathrm{~ms}$ after handover to GNC for liftoff. ${ }^{16}$ The BIMU (an LN200 on the $1.5 \mathrm{~A}$ vehicle) had ceased functioning towards the end of the previous tether test campaign but this was recognized in a Test Readiness Review (TRR) and was not a requirement for flight testing of the otherwise single-string Morpheus flight system. The AFM response to a failed PIMU was to either switch to an available BIMU or perform a soft abort and the latter was attempted. However, the navigation response to a loss of IMU data was to consider the previous acceleration and rotation as still valid until communication could be re-established with the IMU. In this flight, IMU communication was never re-established and the control system requested increasing engine gimbal to counteract a perceived persistent rotation as measured by the last information received from the IMU. This response effectively flipped the vehicle shortly after launch and resulted in a loss of vehicle. 


\section{B. Return to Free Flight}

A subsequent investigation into the loss of vehicle on FF2 was unable to specifically identify where in the chain from PIMU avionics to flight computer the failure occurred. Therefore, a number of robustness modifications including IMU vibe isolation, addition of the SDI500 BIMU as a flight requirement, and hardened redundant connectors from IMUs to flight computer were added to the 1.5 "Bravo" vehicle. Tethered flight testing resumed in May of 2013 with Tether Test 21. During the resumed tether test campaign a JAVAD GPS Real Time Kinematic (RTK) capability was integrated into flight operations and essentially eliminated the GPS position bias observed on the launch pad when in available. However, the navigation system proved susceptible to adjusting back to the uncompensated GPS bias in the event that communication with the RTK base station was lost. Communication robustness with the RTK base station proved variable based on test site electromagnetic interference and the RTK system was not used in flight operations.

No dynamic flight truth measurement source was available for Morpheus navigation, but the surveyed location of the landing pads were available to provide a terminal comparison to the navigation solution. For Free Flights 3 through 5 the navigation solution at touchdown reported landing within $30 \mathrm{~cm}$ of the target sight. Beginning with Free Flight 6 , the actual vehicle location after landing was measured by the operations team to generate an end-offlight comparison to the VTB navigation solution which was generally within $50 \mathrm{~cm}$ of the surveyed location through FF12 (see Table 6).

Table 6. Landing Accuracy $(\mathrm{ND}=$ No Data $)$

\begin{tabular}{|c|c|c|c|c|c|c|}
\hline & \multicolumn{2}{|c|}{ Nav $(\mathrm{m})$} & \multicolumn{2}{c|}{ Measured $(\mathrm{m})$} & \multicolumn{2}{c|}{ Error $(\mathrm{m})$} \\
\hline Flight & east & north & east & north & east & north \\
\hline \hline FF3 & -0.026 & -0.213 & ND & ND & ND & ND \\
\hline FF4 & 0.167 & 0.117 & ND & ND & ND & ND \\
\hline FF5 & -0.173 & -0.128 & ND & ND & ND & ND \\
\hline \hline FF6 & -0.016 & -0.235 & -0.143 & -0.361 & 0.128 & 0.127 \\
\hline FF7 & 0.020 & -0.050 & 0 & 0 & 0.020 & -0.050 \\
\hline FF8 & -0.231 & -0.002 & -0.245 & -0.083 & 0.015 & 0.081 \\
\hline FF9 & 0.032 & -0.165 & 0.240 & -0.213 & -0.209 & 0.048 \\
\hline \hline FF10 & 0.225 & -0.413 & 0.345 & -0.446 & -0.120 & 0.033 \\
\hline FF11 & 0.228 & -0.421 & 0.287 & -0.319 & -0.058 & -0.102 \\
\hline FF12 & 0.082 & -0.397 & -0.019 & -0.258 & 0.100 & -0.138 \\
\hline
\end{tabular}




\section{ALHAT HRN System Description}

The DL velocimetry and LA sensors were included in ALHAT to provide the necessary information to execute a "soft-touch" landing at any location on a planet but were not deemed sufficient for precision landing within $3 \mathrm{~m}$ of a safe landing site designated in flight by the HDS. ${ }^{6}, 12$ Therefore, considerable effort was put into coordinating the formulation of the ALHAT HRN measurement and its processing by the ALHAT Kalman filter. Fundamentally, the HRN measurement provides information of the location of an observed unique feature in the DEM relative to the HDS sensor assembly. This relative information, in turn, allows the ALHAT Kalman filter to update its location with respect to the DEM and land within tolerances of the safe site (also registered by the HDS in the DEM). Initial concepts for the HRN formulation included angles-only measurements to multiple HRN targets, but the landing timeline on the Morpheus vehicle constrained the processing window to 10-12 seconds and led to an implementation that tracked a single HRN target in flight. The final measurement formulation was a vector position of the HRN target with respect to the HDS assembly structural frame and is given by the expression ${ }^{17}$ :

$$
\hat{\boldsymbol{r}}_{F / I L P}^{s}=\hat{\boldsymbol{T}}_{f}^{s} \boldsymbol{T}_{i, m}^{f} \boldsymbol{T}_{i}^{b}\left(\hat{\boldsymbol{q}}_{i, m}^{b}\right) \boldsymbol{T}_{h}^{b} \boldsymbol{y}_{F / H R N, m}^{h}-\hat{\boldsymbol{r}}_{I L P / H R N, m}^{s} .
$$

Where $\left(\hat{)}\right.$ represents estimated values, $\boldsymbol{r}_{I L P / H R N, m}^{s}$ is the position of the ILP frame with respect to the HRN sensor, $\boldsymbol{y}_{F / H R N, m}^{h}$ is the measured position of the feature with respect to the HRN sensor, and the matrices $\boldsymbol{T}$ are rotations between frames, Note that in this formulation there is a tight coupling between estimated attitude knowledge in terms of the attitude of the vehicle with respect to inertial coordinates $\left(\boldsymbol{T}_{i}^{b}\left(\hat{\boldsymbol{q}}_{i, m}^{b}\right)\right)$ and the estimated position of the HRN with respect to the ILP in the DEM $\left(\hat{\boldsymbol{r}}_{I L P / H R N, m}^{s}\right)$. This coupling led to a derived requirement that attitude knowledge of the VTB and ALHAT navigation strings remain within 0.1 degrees of each other to allow for an in-flight nav quality check on the ALHAT nav solution.

Operationally, the HRN measurement was formulated by correlating a sampled image patch of the HRN feature from the initial DEM image within subsequent HDS laser camera image frames. This required steering the HDS sensor gimbal and tracking the expected location of the HRN feature during flight. Therefore, a feedback loop was required where the HDS system was fed ILP relative position and attitude information from the active navigation string. The constraints on processing time during flight were such that the expected location of the HRN feature had to be within approximately $2 \mathrm{~m}$ of the actual feature to be within the searchable correlation window. Note that this requirement effected navigation error growth from the time the DEM was formed, not across the entire flight. 


\section{ALHAT Integration}

A number of challenges were encountered integrating the ALHAT navigation system into the VTB-capable Morpheus flight system. The most significant of these are outlined in Table 7 and are discussed below.

Table 7. ALHAT Integration Challenges

\begin{tabular}{|c|l|}
\hline Issue ID & Issue \\
\hline AI-1 & Translation state maintenance pre-launch \\
\hline AI-2 & Timing delay with SIGI internally filtered data \\
\hline AI-3 & Attitude initialization \\
\hline AI-4 & Time delay of HRN measurements \\
\hline AI-5 & Time delay of GPS measurements from poll-respond interface \\
\hline AI-6 & Laser sensor interaction with the atmosphere heated by Morpheus engine exhaust \\
\hline
\end{tabular}

The pre-launch translation state was maintained (AI-1) via the navigation cross-feed from VTB to ALHAT as discussed previously. By sending the pre-launch VTB navigation state to the ALHAT filter as a measurement prior to lift-off, the two navigation strings were efficiently and effectively synchronized.

The early ALHAT component testing on Tether Tests 16-18 identified up to $120 \mathrm{~ms}$ timing delay (AI-2) between the VTB primary IMU and the onboard ALHAT HDS system IMU (a LN200). After considerable troubleshooting, the root cause of this timing delay was determined to be processing inertial data from the SIGI that had a $0.2 \mathrm{~Hz}$ low pass filter applied to the data. On the $1.5 \mathrm{~A}$ vehicle this delay was addressed by using alternate outputs that did not have this filtering applied. After return-to-flight with the $1.5 \mathrm{~B}$ vehicle, the Morpheus navigation team worked closely with Honeywell to update the SIGI firmware with filtering parameters that only introduced up to $20 \mathrm{~ms}$ timing delay.

As mentioned previously, a gyro-compassing first stage attitude initialization was used for both strings to get local pitch and yaw knowledge. However, the roll estimates from this procedure manifested as much as 10 degrees variability from initialization to initialization. Given the nature of ALHAT HRN measurements which provided a location of the sensor frame with respect to a feature in the target DEM, any deviation in attitude of greater than 0.1 degrees would prevent a successful ALHAT test (AI-3). Therefore, the Draper GIDE ${ }^{10}$ optical sensor was used in a partially automated mode to refine the attitude states of both the VTB and ALHAT navigation strings. The GIDE optical target was mounted on a precision surveyed mounting fixture at a distance of 70 meters from the GP. A laptop at the vehicle launch pad was used to interface with the GIDE sensor and report the lateral observed offset of the GIDE target within the GIDE camera. This offset was verbally communicated to mission operations and entered into the parameter "i-loads" for both strings of navigation 
and GIDE measurements processed until the attitude covariance converged acceptably. The advantage of this approach was that, providing the vehicle was not moved during flight preparations, the attitude state estimates could be "topped-off" shortly before launch to ensure both attitude accuracy and agreement between the VTB and ALHAT nav strings. The GIDE sensor was not used during flight.

The processing demands of the HRN measurement formulation was such that the relative position of the tracked HRN feature with respect to the HDS assembly was delivered to the Morpheus flight computer up to 2 seconds after the laser camera image of the landing site was taken (AI-4). This delay exceeded the Kalman filter software validity window of $200 \mathrm{~ms}$ (the Kalman filter application ran at $5 \mathrm{~Hz}$ ) that had been applied for all other measurements. An additional capability for measurements with times of validity up to 2 seconds was added by way of a ring buffer storing additional state and covariance data in the Kalman filter software.

During flight testing, a significant delay between the VTB and ALHAT navigation strings was observed, in particular during the boost and lateral translation phase of the flights (AI-5). The root cause of this delay was tracked back to the poll-response communication method between the Morpheus flight computer and the Javad GPS. This led to up to $200 \mathrm{~ms}$ of timing delay as the VTB navigation filter would adjust the states propagated on the IMU to the latent GPS position and velocity values. This timing was variable based on start-up synchronization of the GPS sensor and the Morpheus flight computer. Since the ALHAT sensors did not have this timing issue, an apparent translation state phase difference would manifest between the two navigation strings during dynamic flight. The modification of this timing delay would have required significant redesign of the Morpheus GPS sensor interface software and was deemed too intrusive of a change to the stable baseline VTB navigation platform. Therefore, the delay was left as a variable to be considered when processing flight data.

The most significant ALHAT sensor issue that manifested during flight testing was the interaction of the laser sensors with the heated column of air surrounding the Morpheus main engine exhaust plume (AI-6). This interaction proved to be highly dependent on day-of-launch wind direction and magnitude as super-heated air would translate below the trajectory after boost with a trailing wind. The DL proved the most sensitive to this effect which was mitigated by placing the DL optical head on the forward facing propellant tank of the Morpheus lander for Free Flight 15.

\section{VTB-ALHAT Navigation Tests}

As the VTB navigation system and Morpheus flight performance matured, ALHAT integration was carried out incrementally with a series of tether and flight tests as outlined 
in Table 8. The ALHAT system components were individually tested during Tether Tests 16-18 and later on 25-27 and 34. However, because of the limited altitude and velocity of the tethered test configuration, ALHAT navigation was not able to be tested until actually activated during free flights.

Table 8. VTB-ALHAT Navigation Test Flights (all on 1.5B)

\begin{tabular}{|c|c|c|c|l|}
\hline Test & Date & UPP Source & Nav Source & Notes \\
\hline Tether Test 16-18 & $6 / 2013-7 / 2013$ & VTB & VTB & ALHAT component testing \\
\hline Tether Test $25-27$ & $7 / 2013$ & VTB & VTB & Final pre-flight checkouts \\
\hline Tether Test 34 & $3 / 2014$ & VTB & VTB & Tether test at KSC \\
\hline \hline Free Flight 10 & $3 / 2014$ & VTB & VTB & DL polarity issue \\
\hline Free Flight 11 & $4 / 2014$ & ALHAT & VTB & $\begin{array}{l}\text { DL range inhibited, attitude } \\
\text { error }\end{array}$ \\
\hline Free Flight 12 & $4 / 2014$ & ALHAT & VTB & Lost HRN target track \\
\hline \hline Free Flight 13 & $5 / 2014$ & ALHAT & ALHAT & First full ALHAT test \\
\hline Free Flight 14 & $5 / 2014$ & ALHAT & ALHAT & Night Flight \\
\hline Free Flight 15 & $12 / 2014$ & ALHAT & ALHAT & Successful ALHAT test \\
\hline
\end{tabular}

\section{A. ALHAT Open Loop Flight Tests}

Although the VTB and ALHAT navigation strings were developed with the capability to compare results and switch via AFM from ALHAT to VTB in the event of an egregious or persistent navigation error, it was deemed prudent to begin the ALHAT testing free-flights with the ALHAT navigation system running in the background with the VTB system feeding the flight vehicle control loops for FF10-12.

\section{Free Flight 10}

During Free Flight 10 (FF10), several key issues were encountered including: a reverse polarity in the sense of the DL velocimeter beams between the navigation software and the sensor firmware, variable inputs from the DL slant range measurements, and the poll-response GPS related timing issue when compared with the HRN measurements. Nonetheless, the ALHAT DL and LA sensors were shown to function properly over a significant portion of the trajectory and a single in-flight HRN measurement tracking of an HRN target was demonstrated using the VTB navigation as input. The effect of the thermal plume of Morpheus propulsion exhaust can be seen in Figures 15 and 16 with dropouts observed in all three DL beams and significant early dropout of the LA slant range. 

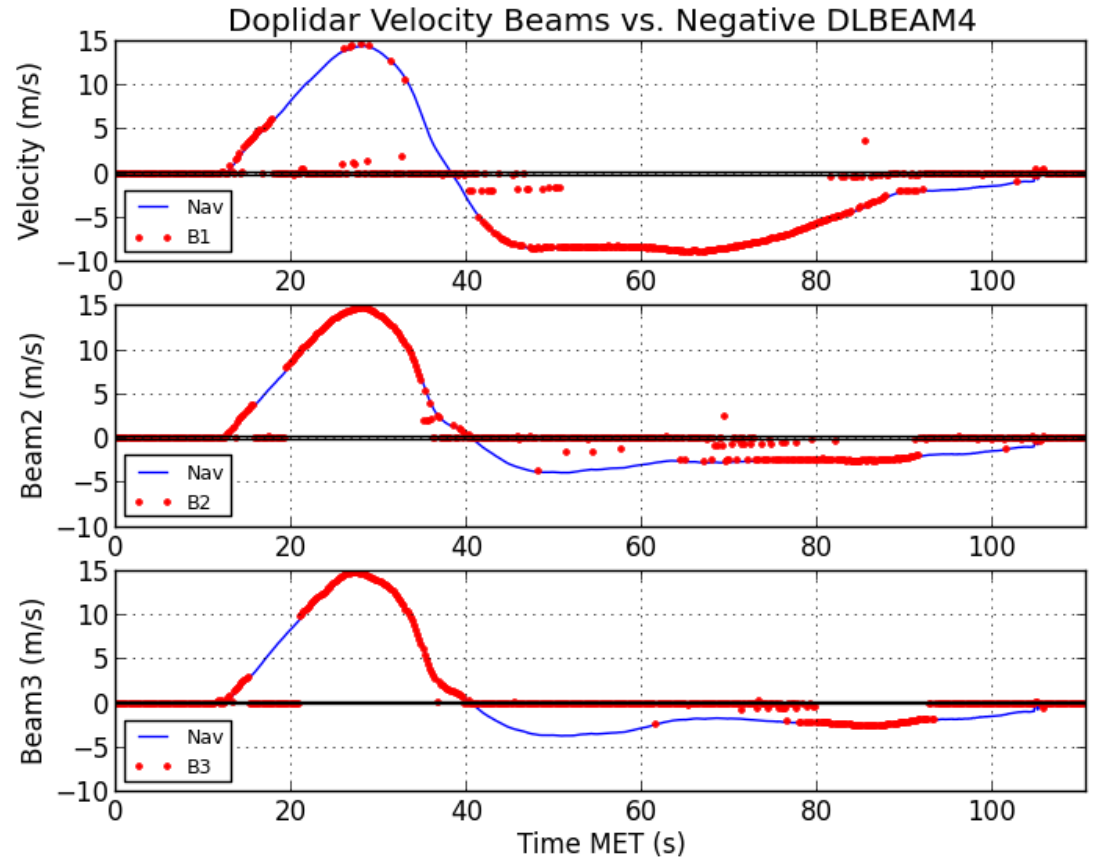

Figure 15. FF10 Doppler Lidar Velocimetry (polarity corrected)

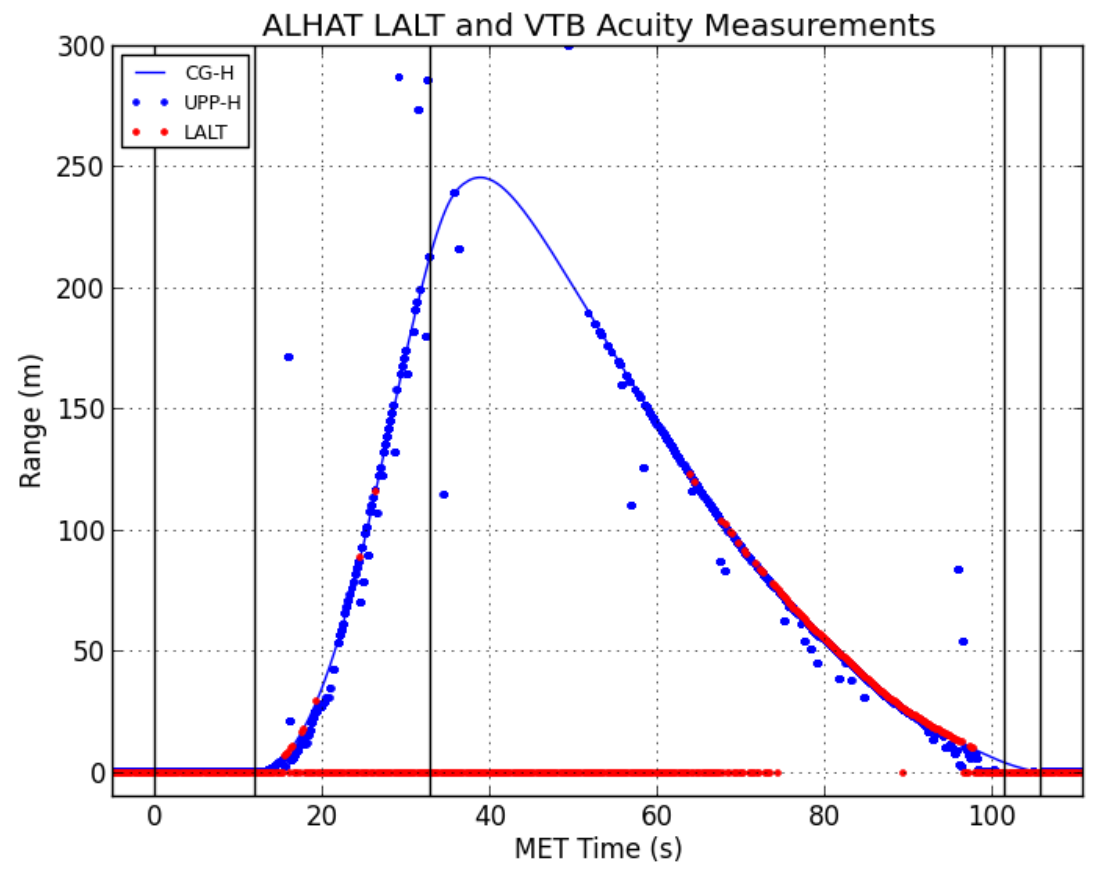

Figure 16. FF10 Laser Altimeter Measurements 


\section{Free Flight 11}

During Free Flight 11 (FF11) the VTB navigation source was used for vehicle flight control but the results of FF10 had given the navigation team confidence that the ALHAT navigation inputs could be used for HDS gimbal control and feature tracking. The DL slant range measurements were inhibited from Kalman filter processing from this flight on to reduce the complexity of the processing and analysis of the ALHAT flight data. LA slant range measurements were more persistent and consistent with the Acuity measurements in FF11. DL velocimetry again showed strong sensitivity to the exhaust plume with significant dropouts in beam 1 and 3 during alternating periods of flight. An operational error on FF11 led to omitting a final GIDE alignment calibration of attitude prior to launch and the "roll" channel covariance was larger than expected. The net result of the larger attitude error and partial DL velocimetry was a nav walk-off that began around MET 10950s (the MET reference time was not reset at ignition for this test). Figures 17 and 18 demonstrate a incurred North velocity bias at this time and a resulting North position error increase over time. However, the benefit of the fully functional DL velocimeter was observed at approximately MET 10967 when all three of the DL beams began reporting measurements. At this time, the velocity errors between navigation strings converged to nearly zero and the position error growth was arrested at approximately 7 meters. A set of four HRN vector measurements were processed on FF11 (see Figure 19) demonstrating the first closed loop control on HDS gimbals and processing of all three ALHAT measurement data types in the ALHAT Kalman filter.

\section{Free Flight 12}

Free Flight 12 demonstrated much the same behavior as FF11 from the LA and DL sensors. The attitude pre-launch synchronization with the GIDE measurement was applied per procedure keeping the roll channel of attitude in alignment between both navigation strings. The HRN only returned a single measurement in FF12 as the navigation solution drifted out of tolerance for the correlation matching of subsequent images. Nonetheless, the test demonstrated sufficient agreement between ALHAT and VTB navigation that the team was prepared to begin closed loop flights, relying on the AFM commanded VTB navigation switchover to manage any HRN processing issues that might arise. 

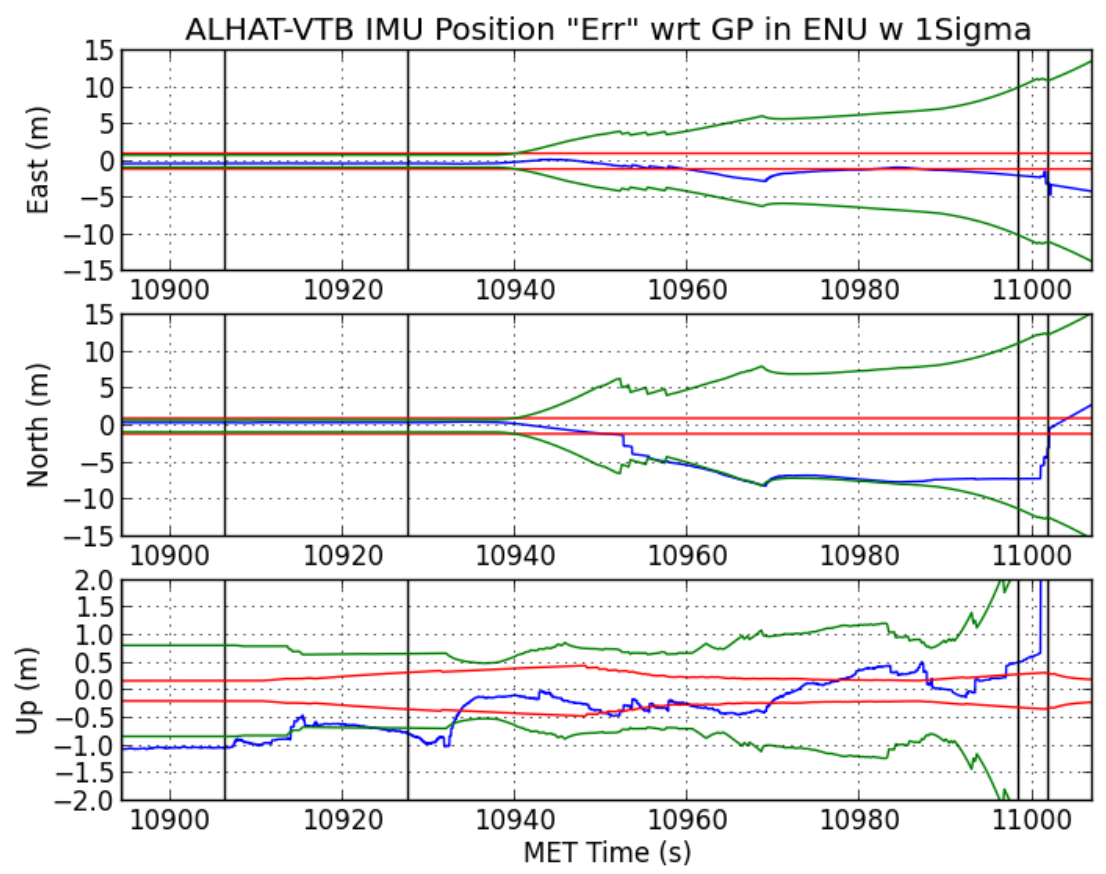

Figure 17. FF11 ALHAT-VTB Position Difference, green=ALHAT filter uncertainty, red $=$ VTB filter uncertainty, blue $=$ ALHAT-VTB difference'
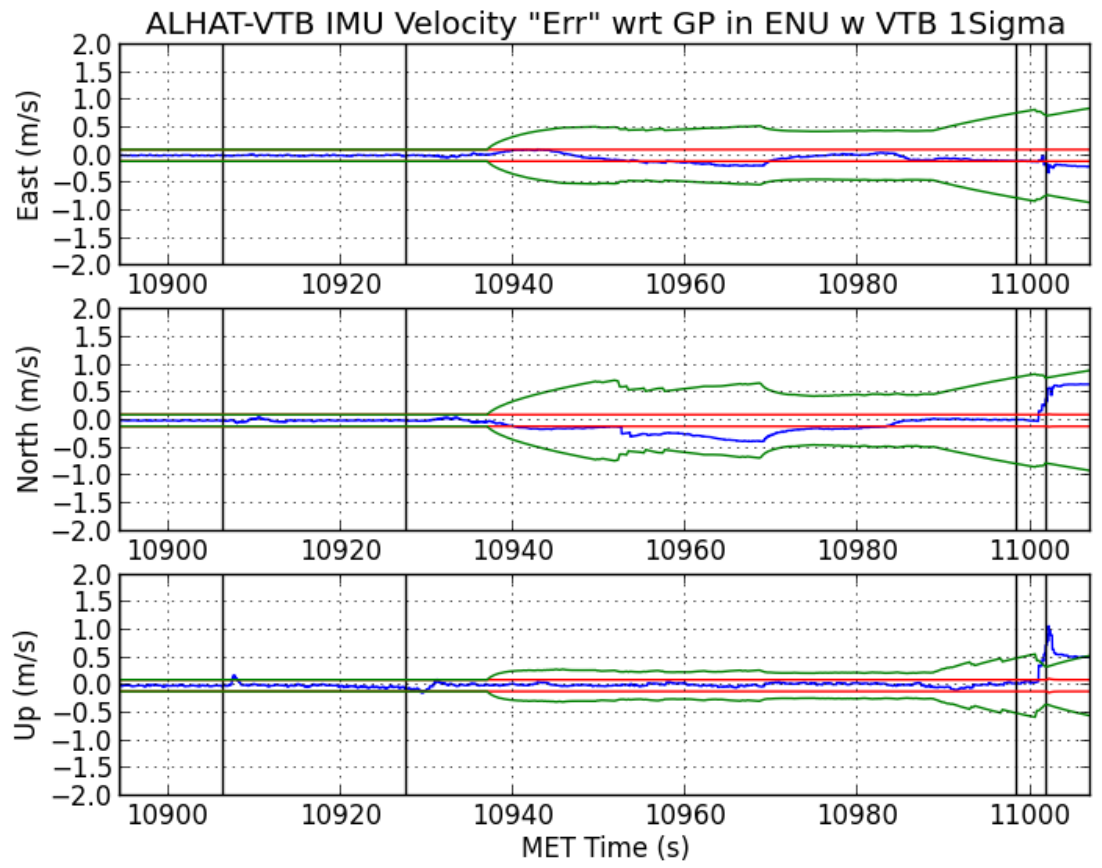

Figure 18. FF11 ALHAT-VTB Velocity Difference, green=ALHAT filter uncertainty, red $=$ VTB filter uncertainty, blue $=$ ALHAT-VTB difference 


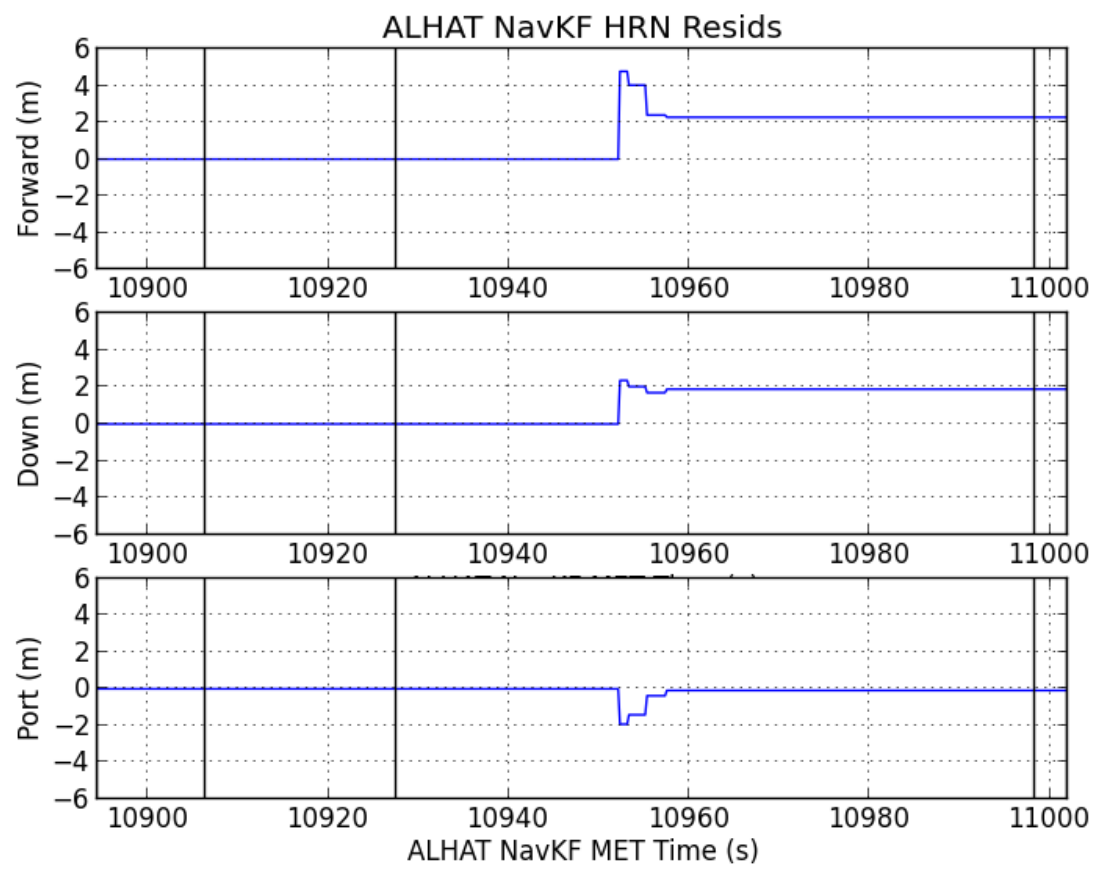

Figure 19. FF11 ALHAT HRN Residuals 


\section{B. ALHAT Closed Loop Flight Tests}

\section{Free Flight 13 - First Closed Loop ALHAT Navigation Test}

Closed loop ALHAT testing began with Free Flight 13 (FF13) and exhibited much the same performance as FF12. The LA and DL sensors were again influenced by the Morpheus exhaust plume, but the team now had enough data of which of the DL beams were effected that it could be correlated to wind direction blowing the heated column of air under the vehicle into the paths of sensing beams. Attitude error was maintained to within tolerances per procedure in FF13. Two sets of vector HRN measurements were processed (Figure 20) but showed a divergent behavior. The overall translation state performance was stable (Figures 21 and 22) but the divergent HRN measurement processing and partial availability of DL velocimetry led to a divergent state comparison outside of the AFM threshold as illustrated in Figure 23. It was enlightening to note that at MET 81 seconds when all 3 beams of the DL velocimeter we available that the position estimates of the VTB and the ALHAT strings of navigation began to converge. A closer examination of the partial derivatives for planet relative velocimetry ${ }^{17}$ revealed a strong correlation between vehicle attitude, velocimetry measurements, and the cross product of vehicle position in the inertial frame when converting the rotating frame measurements into the internal Kalman filter inertial system. It is therefore possible to update the inertial position of the vehicle with sufficiently accurate attitude knowledge when processing DL velocimetry.

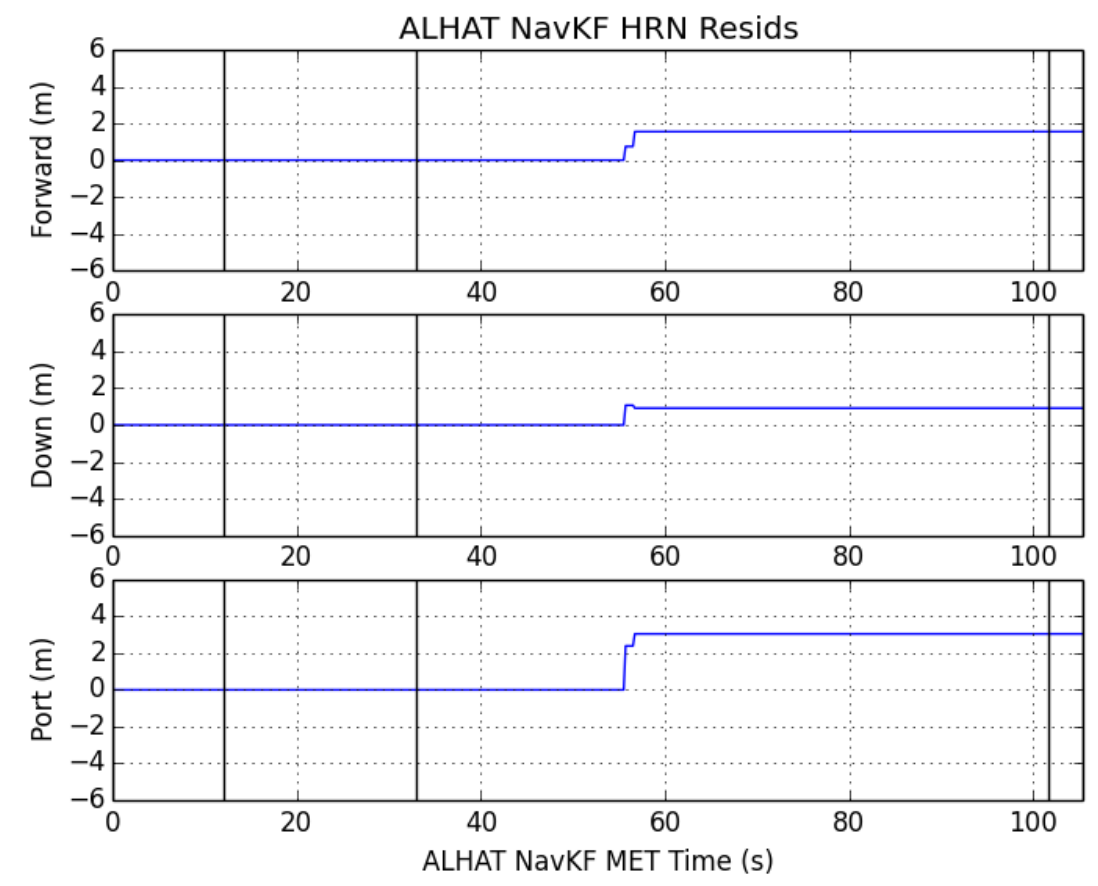

Figure 20. FF13 HRN Processing Residuals 


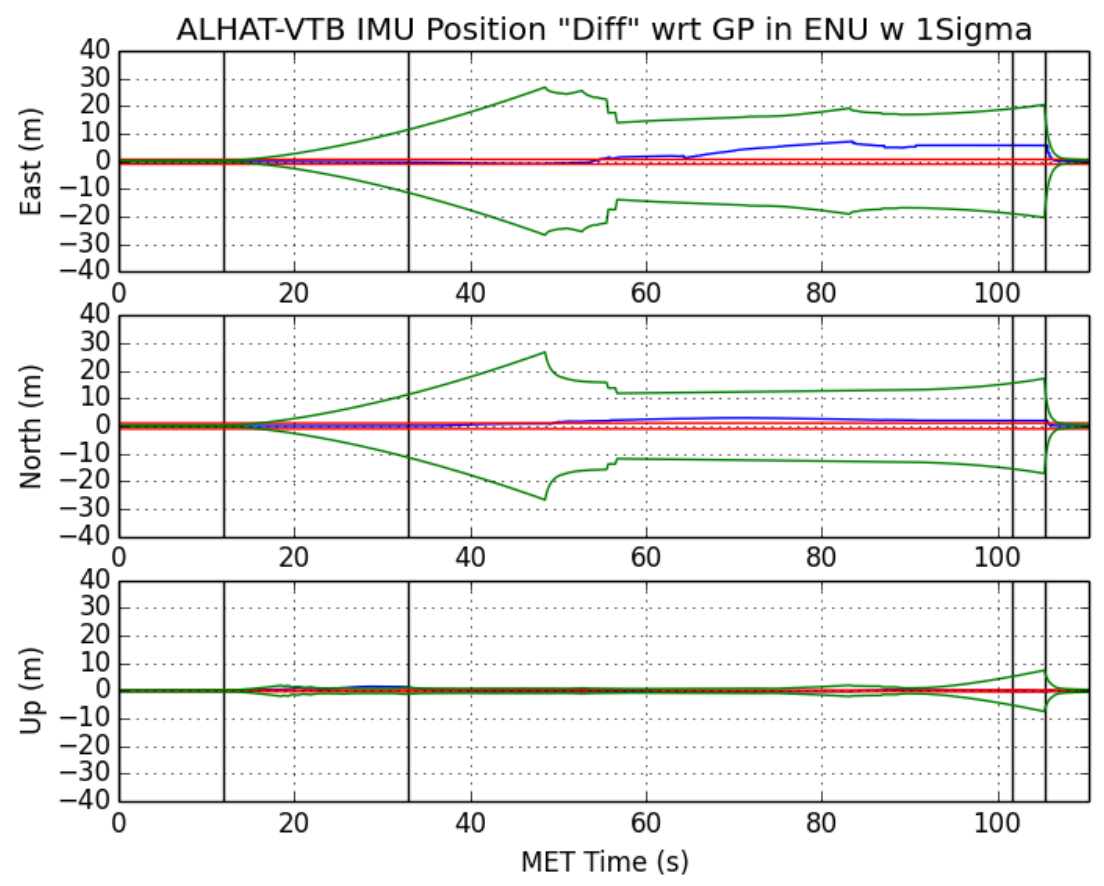

Figure 21. FF13 ALHAT-VTB Position Difference, green=ALHAT filter uncertainty, red $=$ VTB filter uncertainty, blue $=$ ALHAT-VTB difference 

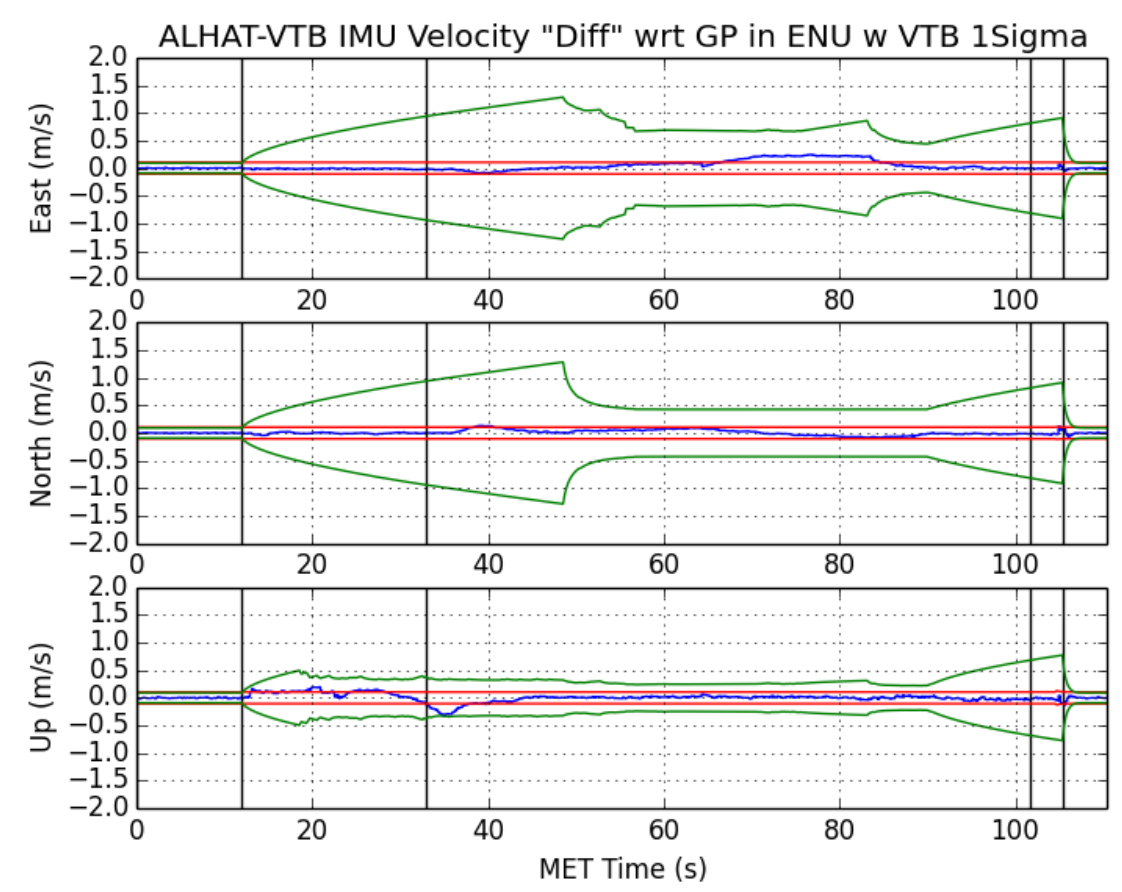

Figure 22. FF13 ALHAT-VTB Velocity Difference, green=ALHAT filter uncertainty, red $=$ VTB filter uncertainty, blue $=$ ALHAT-VTB difference

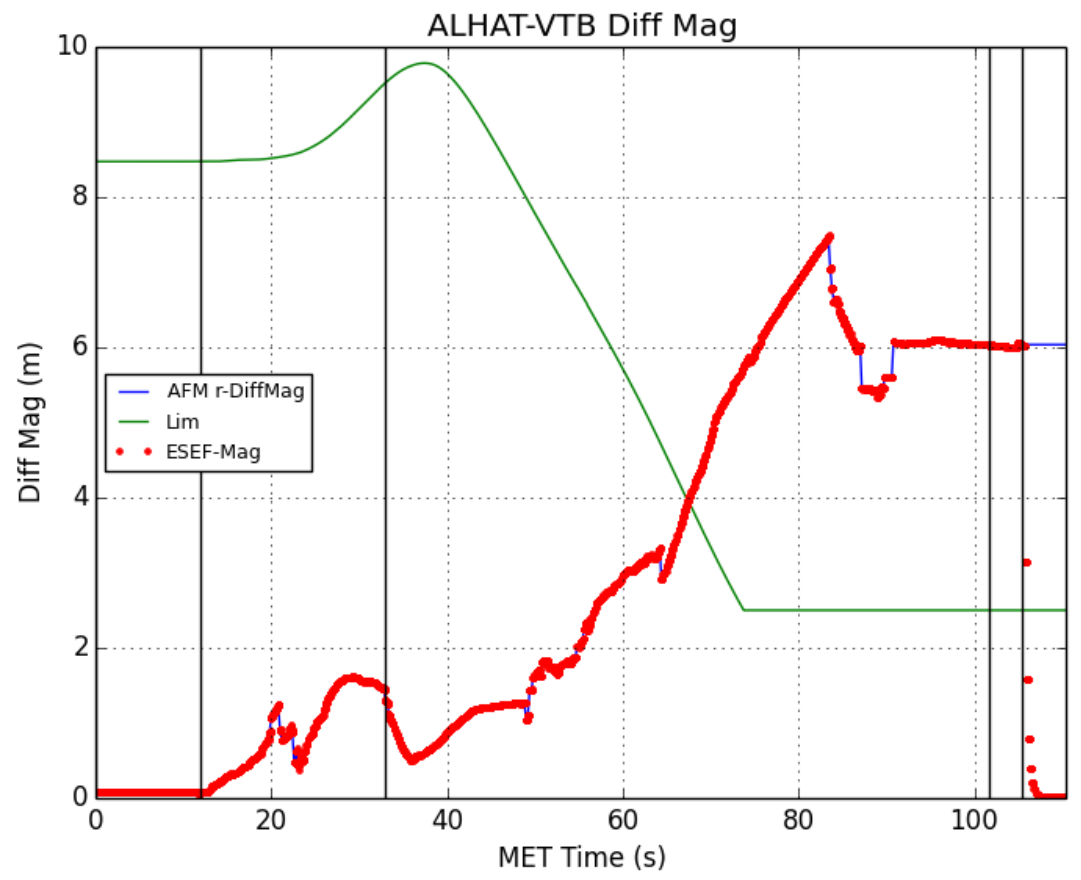

Figure 23. FF13 ALHAT-VTB Position Difference (red) and AFM Switch Threshold (green), downmode from ALHAT to VTB nav source at approx. MET 67s 


\section{Free Flight 14 - Night Flight}

Considerable effort was spent post-processing the FF13 results to determine the cause of navigation divergence when processing the HRN measurements. It was believed that a slight error in the partial derivative formulation in software related to attitude might be the cause and adjustments were made. Also, a modification was put in place to only process DL velocimetry when all three beams were available for processing. FF14 was therefore attempted with the objectives of successfully processing multiple HRN measurements while also demonstrating night-flight operations. The LA and DL sensors were again influenced by the exhaust plume and the attitude error was within tolerances. As with FF13 only two HRN measurements were processed and these also demonstrated divergent behavior. All three DL velocimeter beams came online at approximately MET 70 seconds and pulled the state difference between VTB and ALHAT nearly to within landing tolerances, see Figure 24. However, the 3-beam operations ceased at about MET 90 and the state diverged past landing tolerances with less than 10 seconds to go in the flight sequence.

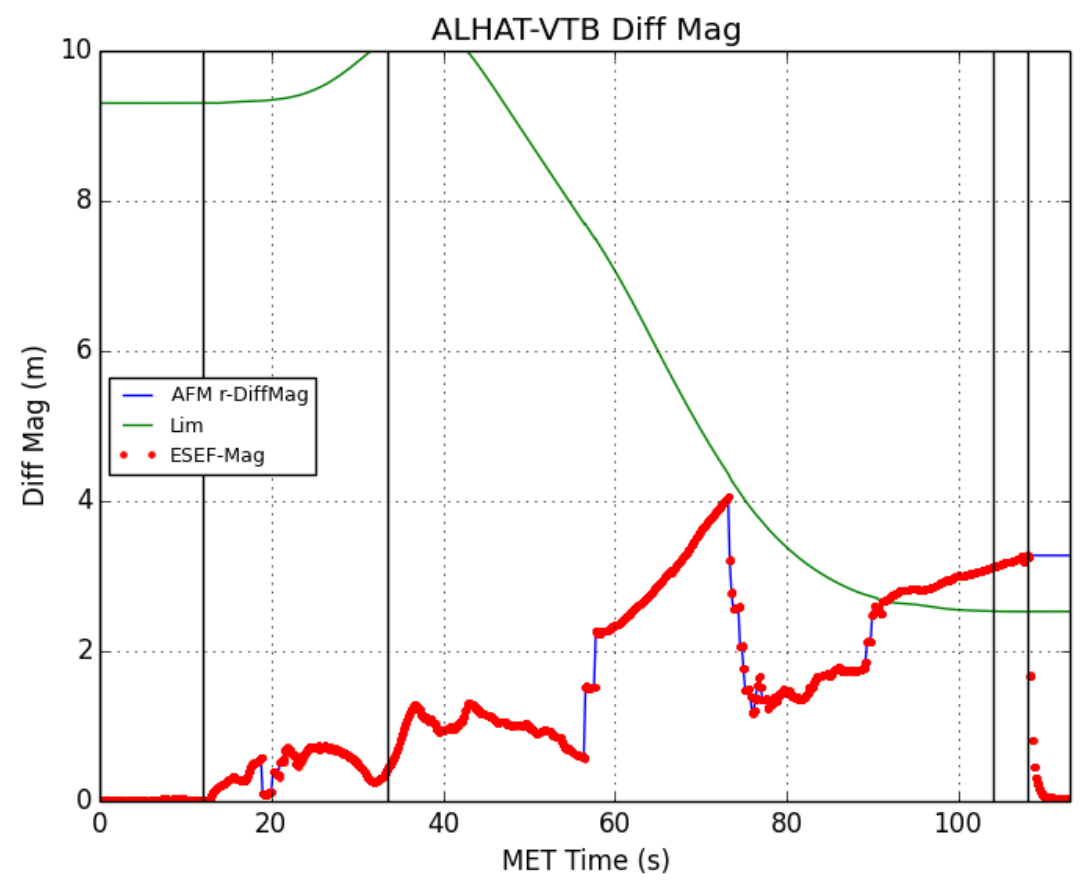

Figure 24. FF14 ALHAT-VTB Position Difference (red) and AFM Switch Threshold (green), downmode from ALHAT to VTB nav source at approx. MET 90s 


\section{Free Flight 15 - Final Flight and ALHAT HRN Success}

The mixed results of DL velocimetry beam availability and HRN measurement processing from FF12 and FF13 prompted the Morpheus/ALHAT project to stand down testing and thoroughly analyze the vast amount of available test data. The ALHAT sensors were inspected by the hardware team and the DL optical head moved to a position higher and on the "forward" propellant tank to orient its beams away from the Morpheus exhaust plume. During this time, two major breakthroughs were realized on the HRN processing: (1) an alternate formulation of the same HRN measurement resulted in less state transition matrix manipulation of the HRN measurement and (2) the feeback loop from the ALHAT navigation string to the HDS processor was found to have a timing issue of significance. The latter proved to be the root cause for measurement divergence because the HDS processor was steering the laser camera to a pre-updated Kalman filter state and returning a measurement. Unfortunately, the Kalman filter had already applied an HRN correction by the time the next measurement was made available and a book-keeping of correction error was realized. This issue was not discovered in simulation because the modeled HDS software did not have an actual processor delay, rather one was synthetically applied. The ALHAT/HDS interface was modified to ensure that measurements and updates were consistent and a simulation modeling both approaches was conducted to show both previous divergent HRN processing and convergent HRN processing with the timing modification.

With these changes in place, FF15 was executed in late 2015 with the potential for a follow-on flight should all ALHAT navigation objectives not be realized. The LA measurements were severely limited in this test, cutting out after $50 \mathrm{~m}$ altitude on the ascent and not resuming measurements until around $100 \mathrm{~m}$ altitude on the descent (Figure 25). However, the DL optical head modifications were very effective and DL velocimetry 3-beam operations were available throughout flight (Figure 26). Attitude errors were maintained as expected (Figure 27). A record $7 \mathrm{HRN}$ vector measurements were processed displaying convergent behavior as demonstrated in Figure 28 until the HDS optics were too close to the hazard field to generate further measurements. The main impact of the HRN updates were on the North and Up channels of navigation (Figures 30 and 31). The apparent difference in the North channel position estimate is also attributed to the GPS timing delay manifesting itself during lateral flight. This hypothesis is bolstered by the convergent behavior of the ALHAT-VTB position difference in Figure 29 where the position differences close after 70 seconds when the lateral translation of the vehicle begins to wind down for descent phase operations. Further detail on the VTB and ALHAT states is available in Figures 32 and 33. 


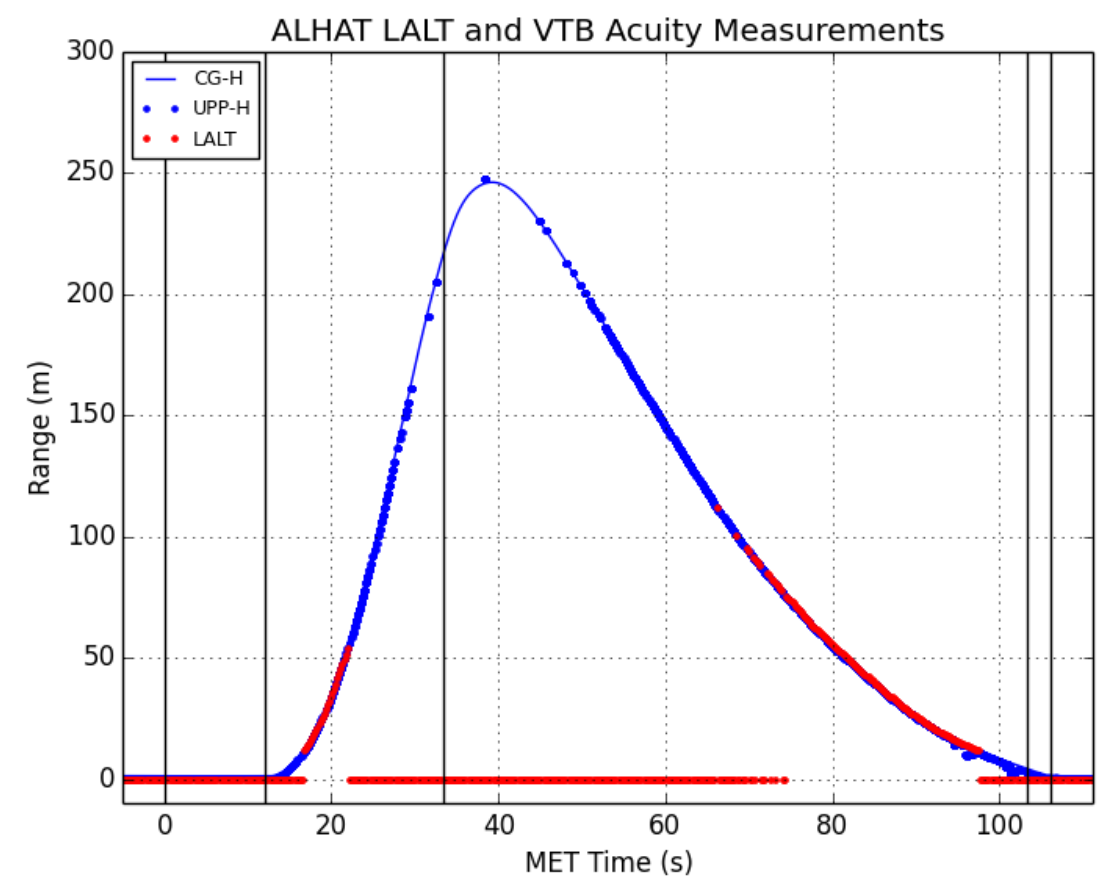

Figure 25. FF15 Laser Altimeter Measurements (red) vs. Acuity Measurements (blue)
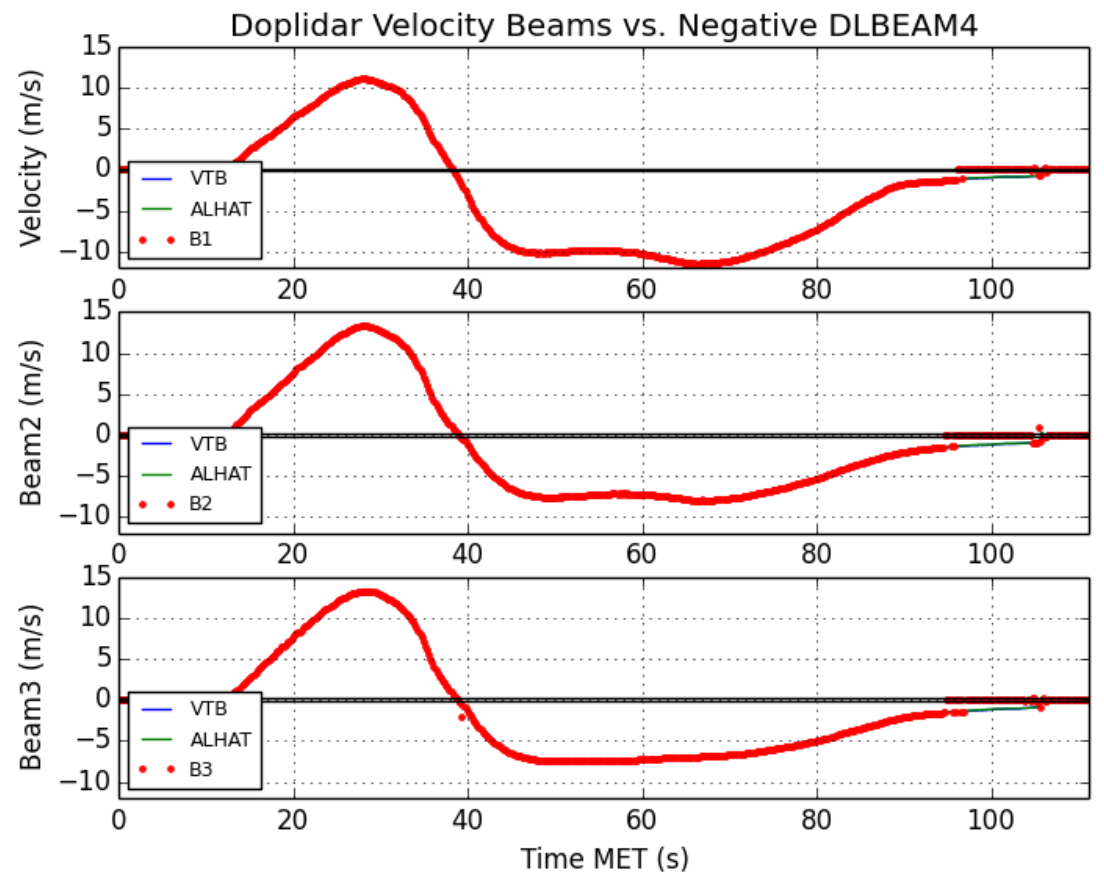

Figure 26. FF15 Doppler Lidar Velocimetry (polarity corrected) 

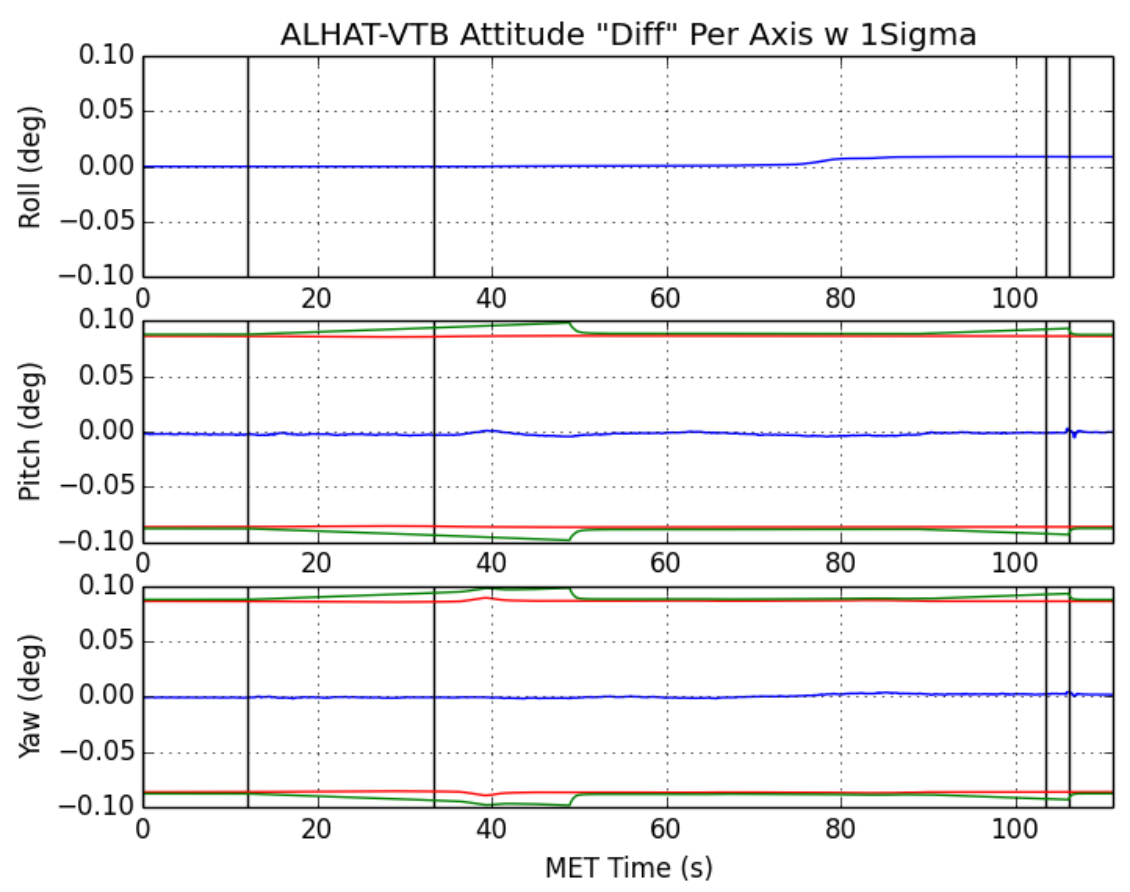

Figure 27. FF15 ALHAT-VTB Euler Angle Difference, green=ALHAT filter uncertainty, red $=$ VTB filter uncertainty, blue $=$ ALHAT-VTB difference

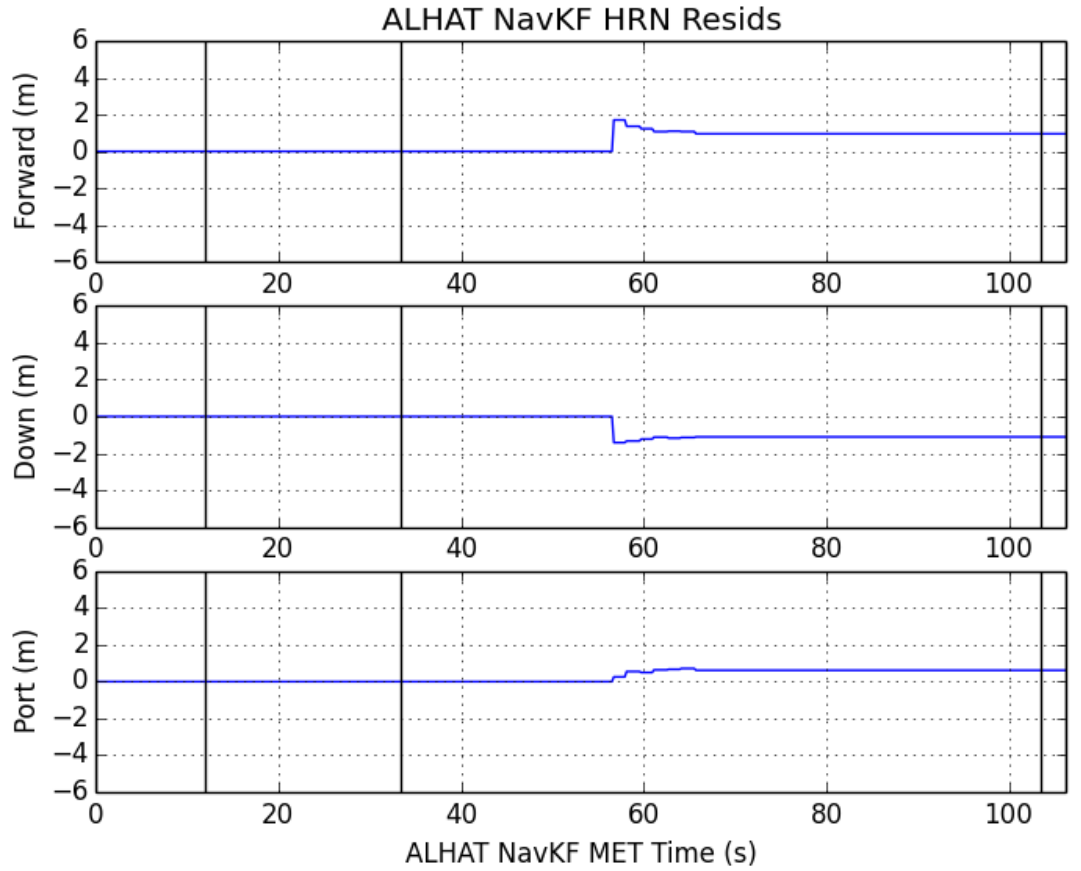

Figure 28. FF15 HRN Processing Residuals 


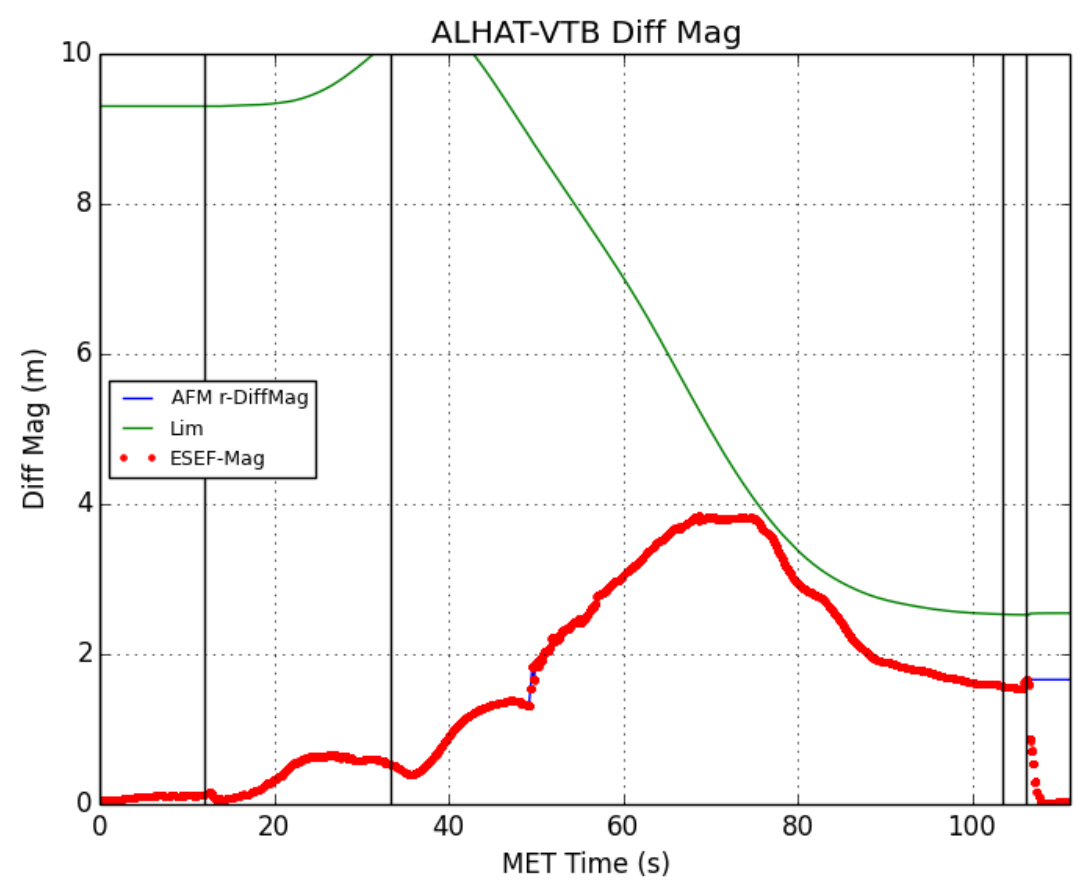

Figure 29. FF15 ALHAT-VTB Position Difference (red) and AFM Switch Threshold (green)

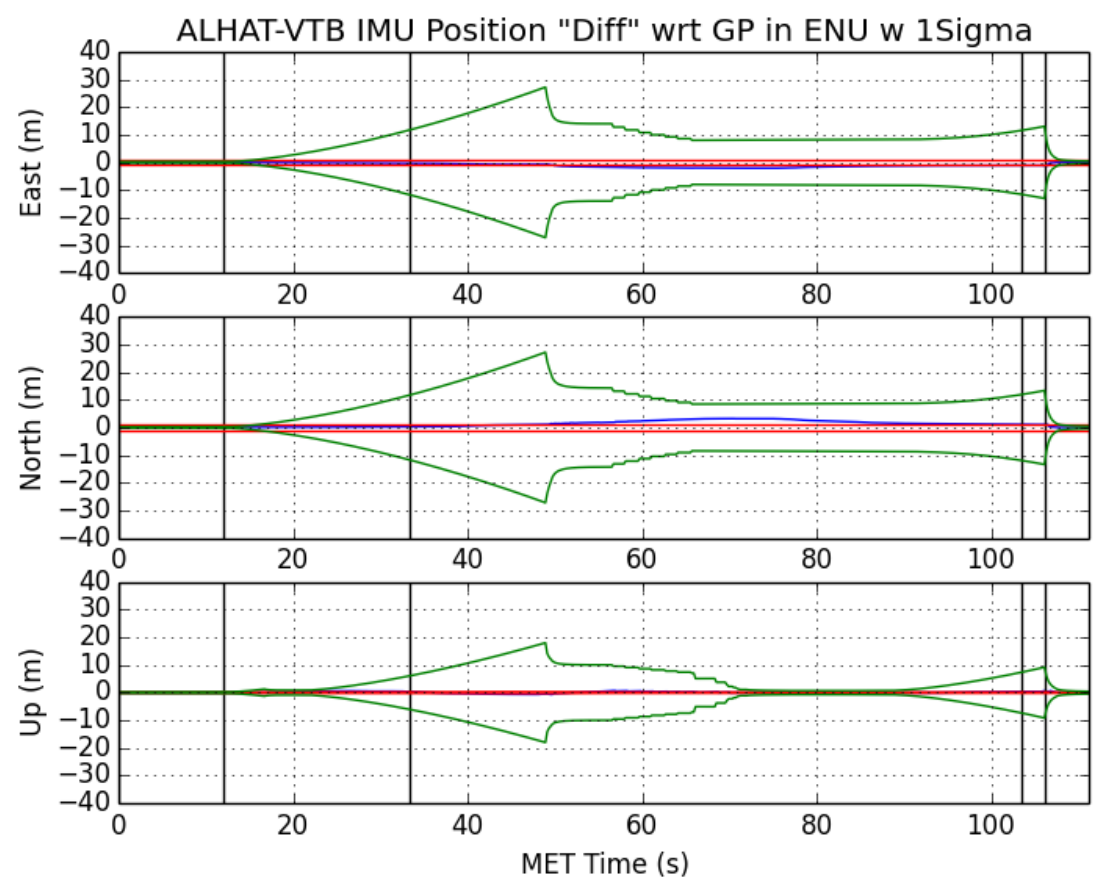

Figure 30. FF15 ALHAT-VTB Position Difference, green=ALHAT filter uncertainty, red $=$ VTB filter uncertainty, blue $=$ ALHAT-VTB difference 

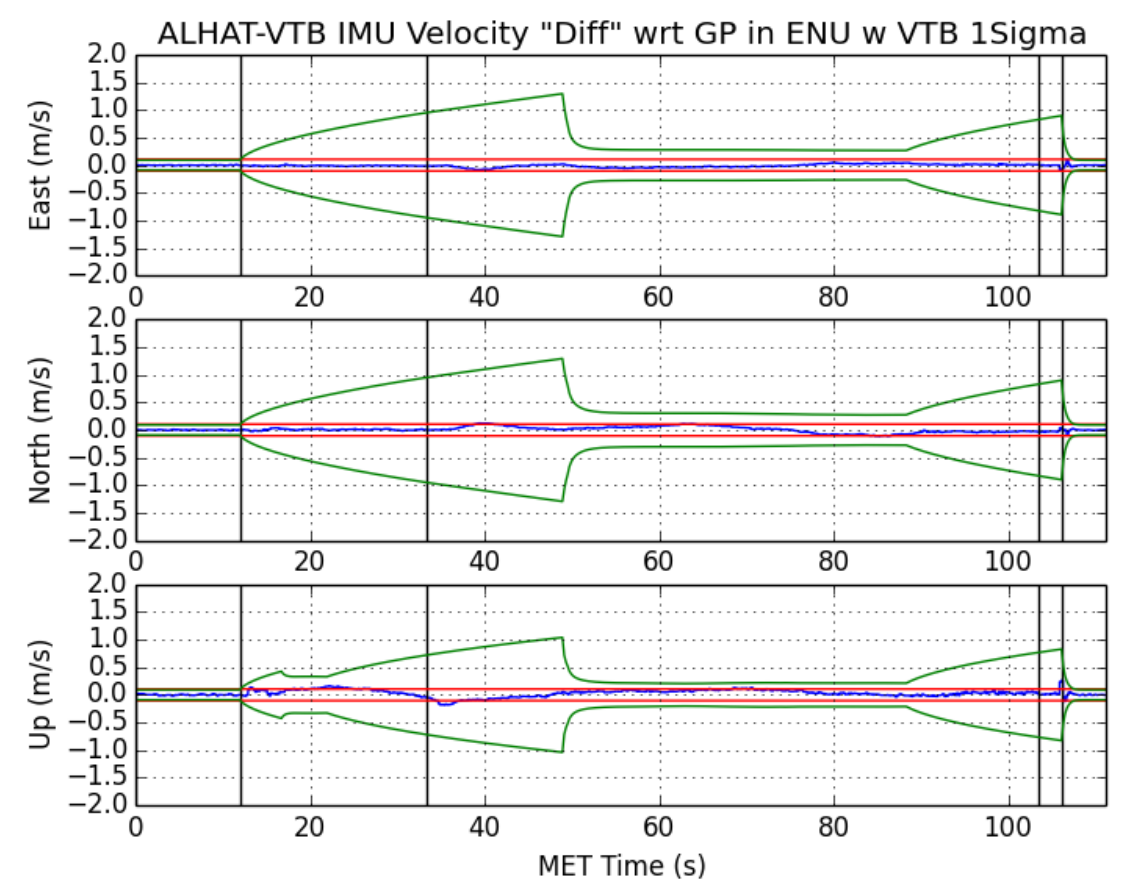

Figure 31. FF15 ALHAT-VTB Velocity Difference, green=ALHAT filter uncertainty, red $=$ VTB filter uncertainty, blue $=$ ALHAT-VTB difference

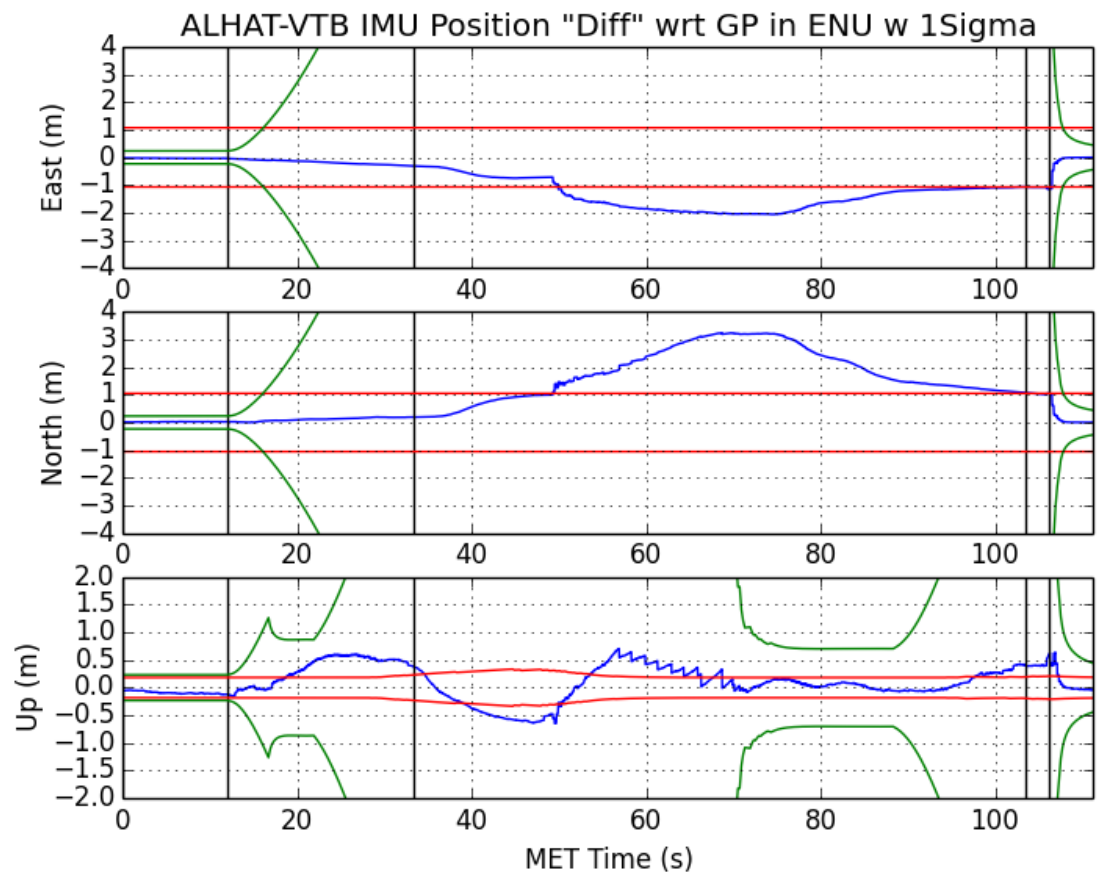

Figure 32. FF15 VTB-ALHAT Nav Position Difference (Zoom), green=ALHAT filter uncertainty, red $=$ VTB filter uncertainty, blue $=$ ALHAT-VTB difference 


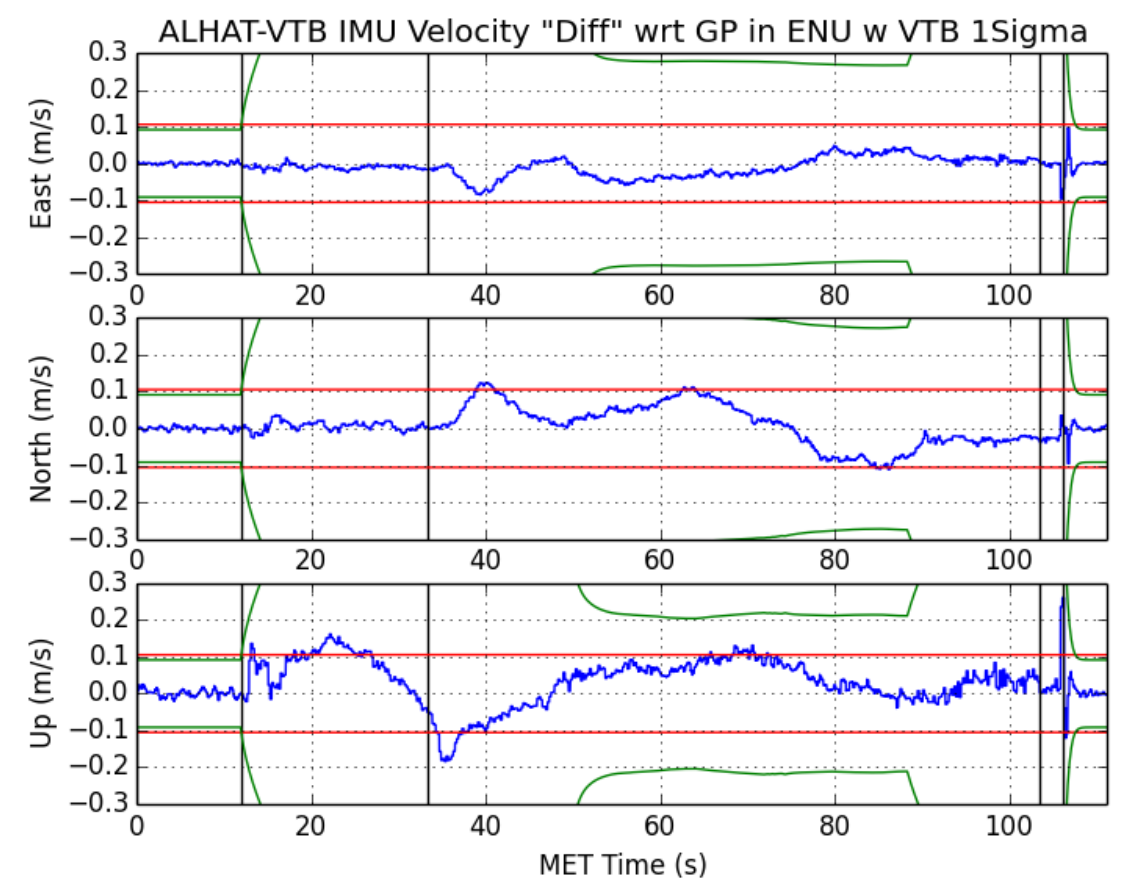

Figure 33. FF15 VTB-ALHAT Nav Velocity Difference (Zoom), green=ALHAT filter uncertainty, red $=$ VTB filter uncertainty, blue $=$ ALHAT-VTB difference 


\section{Summary and Recommendations}

The VTB and ALHAT navigation systems were designed, implemented and field tested over the 4 year interval from late 2010 until late 2014 in concert with the Morpheus Project's demonstration of lean development practices, vehicle automation, and a novel LOX/LCH4 propulsion system ${ }^{11}$. The culmination of this effort from a navigation point of view were the Free Flight tests 13-15 which demonstrated autonomous identification and tracking of an in situ identified target, operation and Kalman filtering of three new surface relative sensor systems (DL, LA, and HRN), night-time operations, and ultimately precision landing with respect to a safe location identified in flight. The navigation system design was derived from lessons learned from the Apollo and Space Shuttle programs with updates for systems such as modern strapdown IMUs implemented in software using a rapid prototyping approach. The enabling aspect of the success of the VTB/ALHAT navigation development was the ready availability of sensor hardware and small-scale field test data. The build-a-little-testa-little paradigm allowed for incremental progress throughout the test campaign in a way that managed acceptable risk. The only limiting factor to this approach was in the ALHAT testing phase was the fact that the ALHAT sensors were typically not fully functional during tethered testing flight envelopes and were only able to be evaluated during full free flights. The VTB and ALHAT navigation systems both performed to within expectations and by FF15 met all requirements (see Table 9 and Figure 34).

Table 9. Landing Accuracy - ALHAT Closed Loop

\begin{tabular}{|c|c|c|c|c||c|c|}
\hline & \multicolumn{2}{|c|}{ Nav } & \multicolumn{2}{c||}{ Measured } & \multicolumn{2}{c|}{ Error } \\
\hline Flight & east & north & east & north & east & north \\
\hline \hline FF13 & 0.01190 & -0.3294 & 0.0327 & -0.1342 & -0.0208 & -0.1951 \\
\hline FF14 & 0.12590 & -0.4114 & 0.2183 & -0.2258 & -0.0924 & -0.1856 \\
\hline \hline FF15 & 1.23710 & -0.9533 & 0.4929 & -1.2779 & 0.7442 & 0.3245 \\
\hline
\end{tabular}

\section{Acknowledgements}

The authors would like to recognize the support of the Morpheus and ALHAT teams who were unfailing in their efforts to put together each lab, field, and flight test. Additionally, the leadership of Morpheus Project Manager Jon Olansen and ALHAT Project Manager Dr. Chirold Epp were essential for the success of demonstrating ALHAT navigation in a flight test environment. 


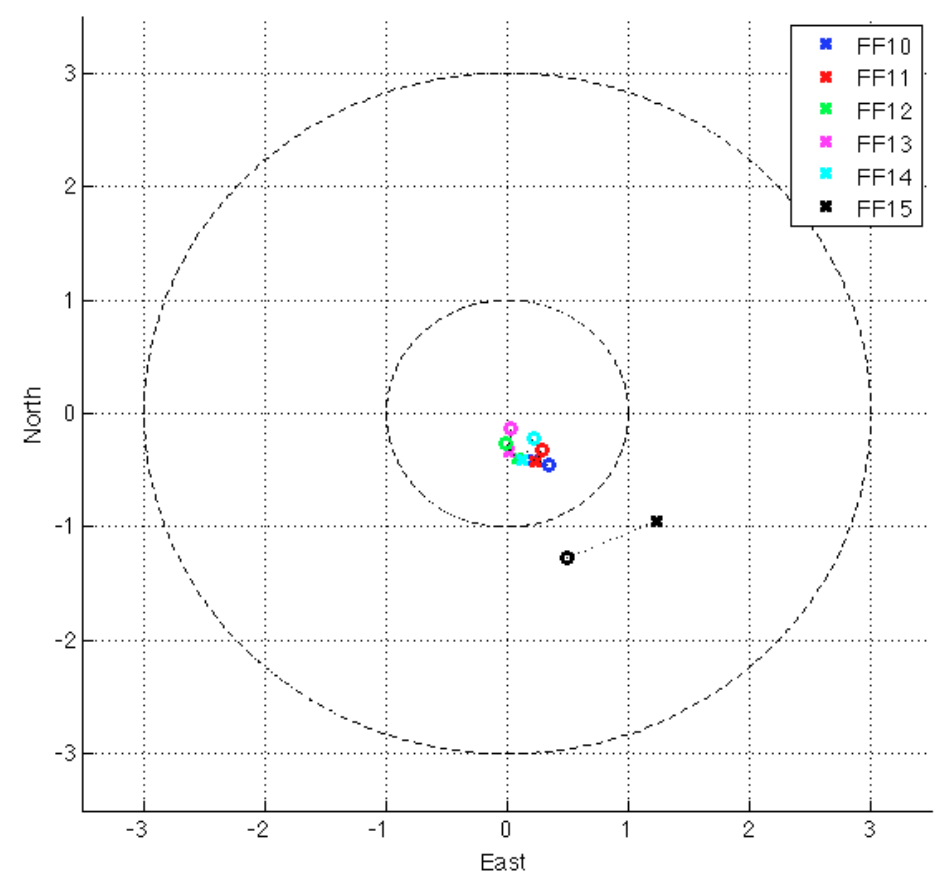

Figure 34. FF11-15 Landing Accuracies (x-nav, o-actual), ALHAT Navigation Accuracy in FF15 


\section{References}

${ }^{1}$ Brady, T., Schwartz, J., and Tillier, C., "System Architechure and Operaterational Concept for an Autonomous Precision Lunar Landing System, 30th Annual AAS Guidance and Control Conference, No. AAS 07-053, 2007.

${ }^{2}$ Epp, C.D. and Smith, T.B., "Autonomous Landing and Hazard Avoidance Technology (ALHAT), in Aerospace Conference, 2007 IEEE, 2007, pp. 1-7.

${ }^{3}$ Epp, C.D., Robertson, E.A., and T. Brady, "Autonomous Landing and Hazard Avoidance Technology (ALHAT), in Aerospace Conference, 2008 IEEE, 2008, pp. 1-7.

${ }^{4}$ Rutishauser, David, Chirold D. Epp, and Edward A. Robertson. "Free-Flight Terrestrial Rocket Lander Demonstration for NASA's Autonomous Landing and Hazard Avoidance Technology (ALHAT) System," Proc. of AIAA SPACE 2012 (2012).

${ }^{5}$ Epp, Chirold D., Robertson, E., and Carson, J., "Real-Time Hazard Detection and Avoidance Demonstration for a Planetary Lander," AIAA SPACE 2014, San Diego, 2014.

${ }^{6}$ Carson, J. M., Robertson, E., Trawny, N., and Amzajerdian, F., "Flight Testing ALHAT Precision Landing Technologies Integrated Onboard the Morpheus Rocket Vehicle", SciTech 2015; 5-9 Jan. 2015; Kissimmee, FL; United States.

${ }^{7}$ Rudolph E. Kalman, "A New Approach to Linear Filtering and Prediction Problems," Transactions of the ASME - Journal of Basic Engineering, Vol. 82, 1960, pp. 35-45.

${ }^{8}$ Epp, C. D., Robertson, E., and Rutishauser, D., "Helicopter Field Testing of NASAs Autonomous Landing and Hazard Avoidance Technology (ALHAT) System fully integrated with the Morpheus Vertical Test Bed Avionics," AIAA Space 2013 Conference; 10-12 Sep. 2013; San Diego, CA; United States.

${ }^{9}$ Markley, F. L., "Attitude Error Representations for Kalman Filtering", Journal of Guidance, Control, and Dynamics, Vol. 26, No. 2 (2003), pp. 311-317.

${ }^{10}$ Steiner, T., Brady, T., "Vision-based Navigation and Hazard Detection for Terrestrial Rocket Approach and Landing, IEEE Aerospace Conference, Big Sky, Montana, 18 March 2014.

${ }^{11}$ Devolites, Jennifer L., and Jon B. Olansen, "Project Morpheus: Lean Development of a Terrestrial Flight Testbed for Maturing NASA Lander Technologies", SciTech 2015; 5-9 Jan. 2015; Kissimmee, FL; United States.

${ }^{12}$ Geller, D., "Linear Covariance Analysis for Lunar Powered Descent and Landing Navigation, 30th Annual AAS Guidance and Control Conference, Breckenridge, CO, United States, No. AAS 07-055, 2007.

${ }^{13}$ DeMars, K. and Bishop, R.H., "Navigation Analysis to Facilitate Precision Descent Navigation for Landing at the Moon," Galveston, Texas, January 2008. 18th AAS/AIAA Space Flight Mechanics Meeting.

${ }^{14}$ Amzajerdian, Farzin, et al. "Lidar Sensors for Autonomous Landing and Hazard Avoidance." AIAA Space Conference Proceedings. 2013.

${ }^{15}$ Olansen, Jon B., S. R. Munday, and J. L. Devolites, "Project Morpheus: Lessons Learned in Lander Technology Development." Proc. AIAA SPACE 2013 Conference \& Exposition, San Diego, CA. 2013.

${ }^{16}$ Devolites, Jennifer L., Jon B. Olansen, and Stephen R. Munday, "Project Morpheus: Morpheus 1.5 A Lander Failure Investigation Results." AIAA SPACE 2013 Conference and Exposition. 2013.

${ }^{17}$ Robert H. Bishop, Crain, T., Hanak, C., DeMars, K, Carson, J., Trawny, N., and Christian, J., "An Inertial Dual-State State Estimator for Precision Planetary Landing with Hazard Detection and Avoidance," AIAA SciTech 2016, San Diego, 2016. 